\title{
Synthesis and biological evaluation of bolaamphiphilic sophorolipids
}

Elisabeth I. P. Delbeke ${ }^{\mathrm{a}, \mathrm{b}}$, Jonas Everaert ${ }^{\mathrm{a}, \mathrm{c}}$, Olivier Lozach ${ }^{\mathrm{d}}$, Tony Le Gall ${ }^{\mathrm{e}, \mathrm{f}}$, Mathieu Berchel ${ }^{\mathrm{d}, \mathrm{e}}$, Tristan Montier $^{\mathrm{e}, \mathrm{f}, \mathrm{g}, \mathrm{h}}$, Paul-Alain Jaffrès ${ }^{\mathrm{d}, \mathrm{e}}$, Petra Rigole ${ }^{\mathrm{i}}$, Tom Coenye ${ }^{\mathrm{i}}$, Martha Brennich ${ }^{\mathrm{j}, \mathrm{k}}$, Niki Baccile, Sophie L. K. W. Roelants ${ }^{\mathrm{c}, \mathrm{m}}$, Wim Soetaert ${ }^{\mathrm{c}, \mathrm{m}}$, Inge N. A. Van Bogaert ${ }^{\mathrm{n}}$, Kevin M. Van Geem ${ }^{\mathrm{b}}$, Christian V. Stevens ${ }^{\text {a* }}$

aSynBioC, Department of Green Chemistry and Technology, Ghent University, Coupure Links 653, 9000 Ghent, Belgium. E-mail: Chris.Stevens@UGent.be

${ }^{b}$ LCT, Department of Materials, Textiles and Chemical Engineering, Ghent University, Technologiepark 914, 9052 Ghent, Belgium.

'InBio, Department of Biotechnology, Ghent University, Coupure Links 653, 9000 Ghent, Belgium.

'Université de Brest, CEMCA, CNRS UMR 6521, IBSAM, 6 avenue Le Gorgeu, 29238 Brest, France.

'IBiSA SynNanoVect platform, IBSAM, Université de Brest, Faculté de médecine Morvan, avenue Camille Desmoulins, 46 rue Félix Le Dantec, CS 51819, 29218 Brest Cedex 2, France.

fUniversité de Brest, INSERM UMR 1078, IBSAM, Faculté de médecine Morvan, avenue Camille Desmoulins, 46 rue Félix Le Dantec, CS 51819, 29218 Brest Cedex 2, France.

${ }^{g}$ CHRU de Brest, Service de Génétique Moléculaire et d'Histocompatibilité, 5 avenue de Maréchal Foch, 29609 Brest cedex, France.

${ }^{h}$ DUMG, Faculté de Médecine et des Sciences de la Santé, 22 avenue Camille Desmoulins, 29328 Brest, France. iLaboratory of Pharmaceutical Microbiology, Ghent University, Ottergemsesteenweg 460, 9000 Ghent, Belgium. ${ }^{j}$ European Molecular Biology Laboratory, Synchrotron Crystallography Group, 71 Avenue des Martyrs, 38042 Grenoble, France.

${ }^{k}$ Institut Laue-Langevin, Grenoble, Beamline D16, Cedex 9, 38042, Grenoble, France.

'Sorbonne Université, UPMC Univ Paris 06, CNRS, Collège de France, Laboratoire de Chimie de la Matière Condensée de Paris, UMR 7574, F-75005 Paris, France.

${ }^{m}$ Bio Base Europe Pilot Plant (BBEU), Rodenhuizenkaai 1, 9042 Ghent (Desteldonk), Belgium.

${ }^{\mathrm{n}}$ Centre for Synthetic Biology, Department of Biotechnology, Ghent University, Coupure Links 653, 9000 Ghent, Belgium.

\begin{abstract}
New synthetic pathways are proposed for the synthesis of a new set of bolaamphiphilic derivatives starting from microbiologically produced sophorolipids. A total set of 43 new derivatives was synthesized via reductive amination of a previously synthesized sophorolipid aldehyde with diamines and primary amines. The new derivatives were evaluated for their antimicrobial activity against Gramnegative and Gram-positive bacteria. Minimum inhibitory concentration (MIC) and minimum bactericidal concentration (MBC) values were determined for the active compounds. Transfection efficiencies were also evaluated for some of the deprotected derivatives via the assessment of their capacity to transfect three different eukaryotic cell lines in vitro. Finally, the self-assembly properties were evaluated for the deprotected derivatives. Antimicrobial activities were mostly observed for the peracetylated mono- or dicationic bolaamphiphiles and only the deprotected monocationic bolaamphiphile with an octadecyl chain on the nitrogen atom was eligible for the evaluation of its transfection properties. Evaluation of the self-assembly properties indicated that the presence of an octadecyl chain was necessary for micelle formation. Both micelle formation and the net charge of the compounds seem to have an influence on the antimicrobial activity and transfection efficiency. These results are promising for use of bolaamphiphilic sophorolipids in medical and self-assembly applications.
\end{abstract}

\section{Keywords}

Sophorolipids, chemical modification, bolaamphiphiles, antimicrobial activity, transfection efficiency, quaternary ammonium salt, self-assembly 


\section{Synopsis}

Sophorolipids, renewable biosurfactants, are used for the synthesis of innovative bolaamphiphilic compounds for application in the medical sector.

\section{Introduction}

Bolaamphiphiles possess not one but two hydrophilic heads, coupled by a hydrophobic linker. Currently, there is a lot of interest in synthetic bolaamphiphiles because of the unusual architectures created by such molecules. ${ }^{1}$ They form monolayer membranes which can organize into micelles, vesicles, nanotubuli, etc. These supramolecular features often arise spontaneously, they are so-called selforganizing structures. The best known natural examples of bolaamphiphiles are the tetraether lipid membranes of Archaea which are able to grow under very extreme temperatures and at very high salt concentrations. In this context bolaamphiphiles likely contribute to the stabilization of membranes. ${ }^{1-2}$ As a result, they can for example be used in drug or nucleic acid delivery applications to form or stabilize vesicles. ${ }^{3-4}$ After all, most liposomes currently used for this purpose face stability problems such as leakage of the content before reaching the target location. ${ }^{5}$ Moreover, bolaamphiphiles can be used as membrane-spanning linkers in biosensors to detect proteins, antibodies, viruses, etc. ${ }^{6}$ They can also serve as channels for ion transport. ${ }^{1}$ Fluorescent bolaamphiphiles can be used as transmembrane probes for lipid imaging to visualize for example ether lipids in the brain. ${ }^{7}$

In view of the growing ecological awareness and the necessity to develop alternatives for fossil resources, the production of amphiphiles from renewable resources is becoming increasingly important. However, among the synthetic bolaamphiphiles which are described in literature, only a few are produced from renewable resources. ${ }^{8}$ These renewable based bolaamphiphiles are mostly fatty acid - for example derived from vernonia and castor oil - and sugar based derivatives. ${ }^{9-19}$ The use of glutamic acid, proline and vitamin $\mathrm{C}$ was also reported for the synthesis of bolaamphiphilic compounds. ${ }^{20-22}$ Examples of naturally occurring bolaamphiphiles are the carotenoid glycoside crocin $\mathbf{1}$, present in saffron up to $25 \%$, and sophorolipid acid 2 (Figure 1) ${ }^{23-25}$ Sophorolipids are a class of glycolipid biosurfactants which are produced by different yeast species, mainly Starmerella bombicola, from renewable resources. They consist of a hydrophilic sophorose head and a hydrophobic fatty acid tail. The main sophorolipid fermentation products are C18:1 sophorolipid acid $\mathbf{2}$ and diacetylated C18:1 sophorolipid lactone $\mathbf{3}$ which both include oleic acid in their structure (Figure 1). Natural sophorolipids feature interesting biological activities such as anti-cancer, antimicrobial, dermatological, immunoregulatory, spermicidal and antiviral activity. ${ }^{26-27}$ Moreover, they possess self-assembly properties with a high variety in the type of nanostructures formed from different sophorolipid derivatives. ${ }^{28-32}$ For example, C18:1 sophorolipid acid 2 forms micelles with the charge dependent on the $\mathrm{pH}$, while its saturated derivative forms nanoscale ribbons with supramolecular chirality. ${ }^{31}$ Recently, the fermentative production of sophorolipid based bolaamphiphiles with two sugar heads was also reported. ${ }^{33-34}$

In this work, the synthesis of a new class of bolaamphiphilic sophorolipids is described starting from the major microbial fermentation product, i.e. diacetylated sophorolipid lactone 3. A modification pathway previously developed in our group is used for the synthesis of an intermediate sophorolipid aldehyde $\mathbf{6}$ (Scheme 1). ${ }^{35}$ This aldehyde intermediate is then further modified towards different nitrogen containing bolaamphiphilic sophorolipids. With this procedure, the length of the linker can be customized and optimized, which is not the case for derivatives produced via fermentation. The new bolaamphiphilic derivatives were evaluated regarding their antimicrobial, transfection and selfassembly properties.

Previously synthesized quaternary ammonium sophorolipids proved to have good antimicrobial and transfection properties when an octadecyl chain was present on the nitrogen atom. ${ }^{35-36}$ With the described reaction procedures, an overall yield of $23 \%$ was obtained for the quaternary ammonium sophorolipids 
with the ozonolysis and reductive amination being the crucial steps. ${ }^{37}$ If the yields of these reaction steps could be heightened towards $90 \%$ in an industrial setting by avoiding automated chromatography purification, the overall yield of the reaction pathway could be raised to $71 \%$. The carbon efficiency and atom economy were respectively $69 \%$ and $62 \%$ for the sophorolipid aldehyde 3 and respectively $70 \%$ and $48 \%$ for the quaternary ammonium sophorolipids. The valorization of the methyl 9 -oxononanoate byproduct resulting from the ozonolysis step is taken into account. For all reaction steps, the use of green solvents was aimed at, resulting in the replacement of dichloromethane with methanol for the ozonolysis step. $^{38}$
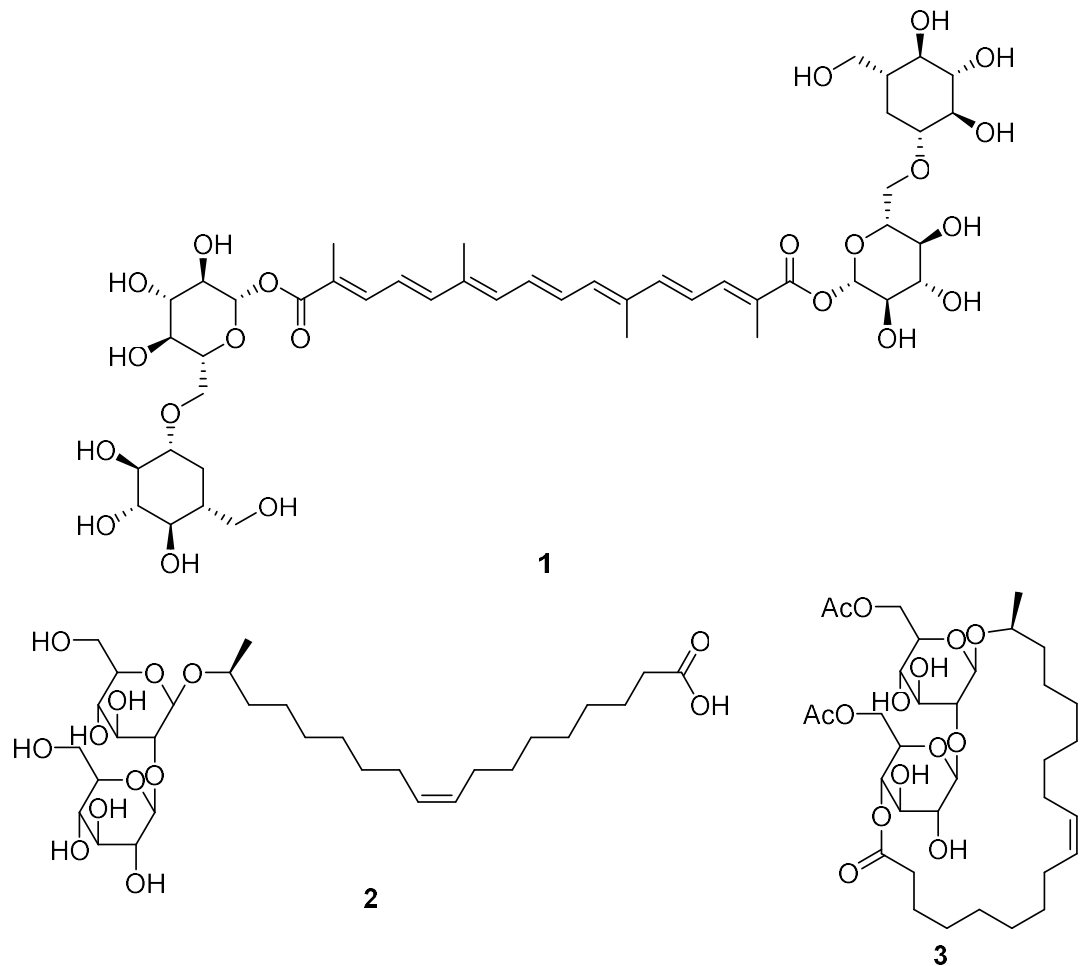

Figure 1. Crocin 1, sophorolipid acid 2 and sophorolipid lactone 3
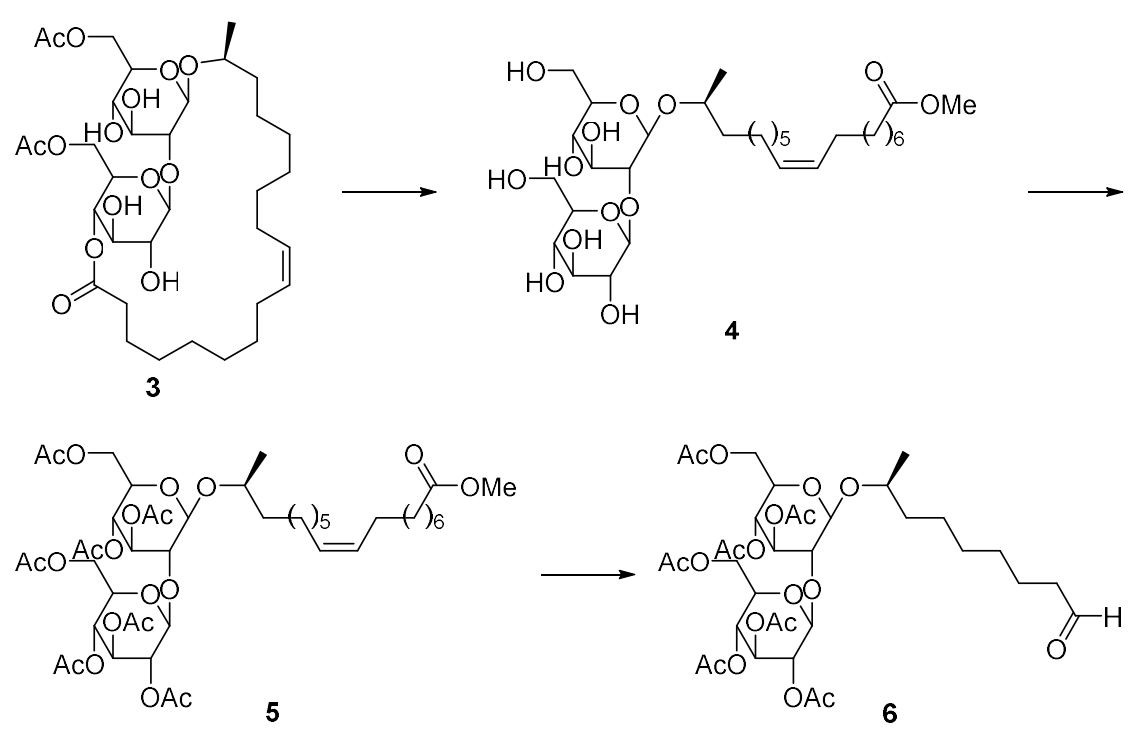

Scheme 1. Modification pathway towards sophorolipid aldehyde 6 


\section{Experimental section}

Determination of the antimicrobial activity. Antimicrobial activity of all bolaamphiphilic sophorolipids against E. coli LMG 8063, K. pneumoniae LMG 2095, P. aeruginosa PAO1, S. aureus ATCC 6538 and S. aureus Mu50 was assessed by a broth microdilution method (CLSI, 2012). ${ }^{39}$ Strains with LMG designation were obtained from the BCCM/LMG Bacteria Collection (Ghent, Belgium), while strain ATCC 6538 was obtained from the American Type Culture Collection (Manassas, VA). S. aureus strain Mu50 was a kind gift of P. Vandamme (Ghent, Belgium). All strains were grown aerobically at $37{ }^{\circ} \mathrm{C}$ on Mueller Hinton agar (LabM, Heywood, UK). The minimal inhibitory concentration (MIC) that inhibited growth completely compared to the untreated control and the minimal bactericidal concentration $(\mathrm{MBC})$ at which no surviving organisms can be recovered were used as a measure of activity. MIC and MBC values were determined using flat-bottomed 96-well microtiter plates (TPP, Trasadingen, Switzerland). Concentrations of compounds tested ranged from 1.22 to $2500 \mu \mathrm{g} / \mathrm{mL}$ in Mueller Hinton Broth (LabM). The inoculum was standardized at approximately $5 \times 10^{4}$ colony forming units $/ \mathrm{mL}$. The plates were incubated at $37{ }^{\circ} \mathrm{C}$ for $24 \mathrm{~h}$, and the optical density was determined at 590 $\mathrm{nm}$ using a multilabel microtiter plate reader (Envision Xcite, PerkinElmer LAS, Waltham, MA).

Liposome formulation. The liposomal solutions were prepared by the lipid film hydration method. A $1.5 \mathrm{mM}$ solution (in $1 \mathrm{~mL}$ ) of each compound was prepared in chloroform, formulated with or without DOPE (1:1 compound/DOPE) and evaporated under reduced pressure to produce a thin lipid film. Water $(1 \mathrm{~mL})$ was added to rehydrate this lipid film in a time period of 7 days at room temperature. The solution was vortexed $(10 \mathrm{~s})$ and sonicated $\left(30 \mathrm{~min}\right.$ at $\left.50^{\circ} \mathrm{C}\right)$ at $45 \mathrm{kHz}$ using a VWR ultrasonic bath. The size and zeta potential were determined for each liposomal formulation.

DNA complexation. Lipoplexes were prepared by mixing pDNA (pEGFP-Luc, Clontech) with each liposomal solution in OptiMEM (Gibco). Addition of pDNA to the liposomal solutions was performed at concentrations corresponding to $\mathrm{CR}$ ranging from 1 to 4 . The obtained mixtures were incubated at room temperature for $1 \mathrm{~h}$ before being subjected to electrophoresis in a $0.8 \%$ agarose gel at $100 \mathrm{~V}, 90$ $\mathrm{mA}$. The gel was stained with SYBRgold nucleic acid gel staining (Life Technologies) and visualized under UV light using a UV trans-illuminator (Fischer Bioblock).

Transfection efficiency. The in vitro reporter gene assay via luciferase measurement was carried out as reported previously. ${ }^{40-41}$ Data were expressed as relative light units (RLU) per milligram of total proteins (means $\pm \mathrm{SD}$ with $\mathrm{n}=3$ ). Lipofectamine 3000 (Invitrogene) was used as standard.

Cell viability. The Vialight kit (Lonza) was used to estimate the viability of the cells following transfection. For this purpose, $48 \mathrm{~h}$ after exposition to the lipoplexes to evaluate, cells were lysed and their ATP content was determined, as recommended by the manufacturer. Non-transfected cells were used to express viability results in the form of percentages ( $\%$ of the reference).

Small Angle X-ray Scattering (SAXS). SAXS experiments are performed at $25^{\circ} \mathrm{C}$ immediately after sample preparation on the BioSAXS BM29 beamline at the ESRF synchrotron facility (Grenoble, France) using $12.5 \mathrm{keV}$ energy and a sample-to-detector distance of $2.867 \mathrm{~m}$, imposed by the beamline standard configuration. The energy is calibrated by measuring the $\mathrm{L}_{\mathrm{I}}$ and $\mathrm{L}_{\mathrm{III}}$ edges of platinum and the sample-to-detector distance is determined using silver behenate $\left(\mathrm{d}_{\text {ref }}=58.38 \AA\right.$ ). (http://www.esrf.eu/home/UsersAndScience/Experiments/MX/About_our_beamlines/bm29.html). ${ }^{42}$

For this experiment, the automatic samples changer for liquids was employed using the 96-well plates and about $100 \mu \mathrm{L}$ of each sample. ${ }^{43}$ The liquid sample is automatically loaded into a $1.8 \mathrm{~mm}$ quartz glass capillary and ten acquisitions of $1 \mathrm{~s}$ each are taken as the sample passes the beam. Individual 
frames are manually controlled for systematic changes and averaged for better statistics if none are found. Eventual changes can be either due to intrinsic sample heterogeneity or radiation damage. The signal of the Pilatus 1M 2D detector, used to record the data, is integrated azimuthally with PyFAI to obtain the $I(q)$ vs. $q$ spectrum $\left(q=\frac{4 \pi}{\lambda} \sin \theta\right.$, where $2 \theta$ is the scattering angle) after masking systematically wrong pixels and the beam stop shadow. ${ }^{44}$ Absolute intensity units were determined by measuring the scattering signal of water $\left(0.0163 \mathrm{~cm}^{-1}\right)$. Radii of gyration, $\mathrm{R}_{\mathrm{g}}$, have been calculated by the Guinier analysis of the SAXS data using the SasView software, available free of charge at the developer's website (http://www.sasview.org).

\section{Results and discussion}

\section{Synthesis of the new derivatives}

A first set of bolaamphiphilic sophorolipid derivatives was synthesized via reductive amination of sophorolipid aldehyde 6 with several diamines. A set of secondary diamines 7 with an ethylene-, hexamethylene, or $o$-phenylenelinker and methyl, butyl or octadecyl groups was selected for the synthesis of the desired bolaamphiphilic sophorolipids (Figure 2). With this set of amines, it was envisioned to assess the influence of the length of the alkyl linker, the presence of an aromatic group in the linker and the length of the substituents on the properties of the derivatives. Since only $N, N^{\prime}$-dimethylethylenediamine $\mathbf{7 a}$ and $N, N^{\prime}$-dimethylhexamethylenediamine $\mathbf{7 b}$ are commercially available, other diamines had to be synthesized.

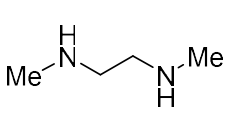

$7 a$<smiles>CNCCCCCNC</smiles>

$7 \mathrm{~b}$

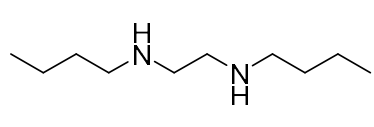

$7 c$

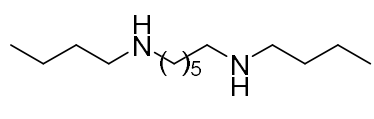

$7 d$

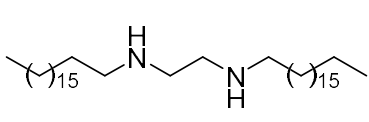

$7 e$

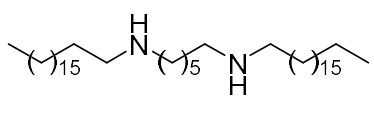

$7 f$
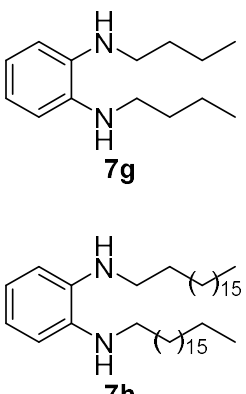

Figure 2. $N, N$-dialkyldiamines 7 as substrates for the reductive amination towards bolaamphiphilic sophorolipids

The intermediate diamides $\mathbf{1 0}$ were synthesized via a Schotten-Bauman reaction with acid chlorides $\mathbf{8}$ and diamines 9 (Scheme 2). Subsequently, these intermediate diamides were reduced with a borane tetrahydrofuran complex towards the desired $N, N^{\prime}$-dialkyldiamines 7 . With this procedure, a total set of eight different $N, N^{\prime}$-dialkyldiamines 7 was available, including the commercially available $N, N^{\prime}$-dimethylethylenediamine $\mathbf{7 a}$ and $N, N$ '-dimethylhexamethylenediamine $\mathbf{7 b}$. These intermediate diamines were not fully characterized and therefore reaction yields were not determined.

The synthesis of the desired bolaamphiphilic sophorolipids 11 was performed according to the reaction conditions described for the previously synthesized sophorolipid amines (Scheme 2). ${ }^{35}$ The reductive amination of sophorolipid aldehyde $\mathbf{6}$ with $N, N^{\prime}$-dialkyldiamines 7a-d resulted in the synthesis of the desired bolaamphiphilic sophorolipids 11a-d. However, reductive amination with $N, N$ '-dialkyldiamines $\mathbf{7 e - h}$ was not successful. In the case of $N, N^{\prime}$-dialkyldiamines $\mathbf{7 e}, \mathbf{7} \mathbf{f}$ and $\mathbf{7 h}$, poor solubility of the diamines in the reaction solvent prevented the synthesis of the desired bolaamphiphilic sophorolipids. When tetrahydrofuran was evaluated as solvent instead of methanol, no reductive amination occurred either. In the case of $N, N$ '-dialkyldiamine $7 \mathbf{g}$, NMR-analysis indicated that the reductive amination did occur partially, but no complete conversion could be obtained due to the steric hindrance caused by the close proximity of the two butyl groups. 

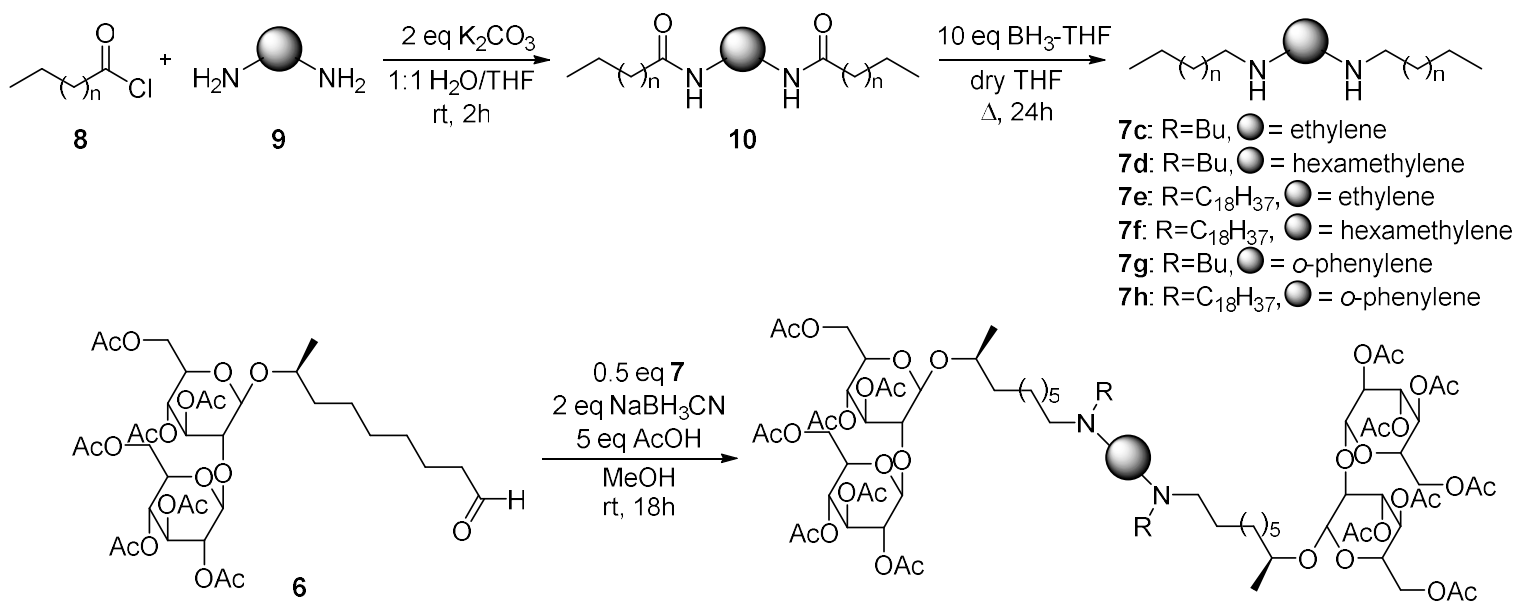

$$
\begin{aligned}
& \text { 11a: } \mathrm{R}=\mathrm{Me}, \mathrm{O}=\text { ethylene }(31 \%) \\
& \text { 11b: } \mathrm{R}=\mathrm{Me}, \mathrm{O}=\text { hexamethylene }(31 \%) \\
& \text { 11c: } \mathrm{R}=\mathrm{Bu}, \mathrm{O}=\text { ethylene }(41 \%) \\
& \text { 11d: } \mathrm{R}=\mathrm{Bu}, \mathrm{O}=\text { hexamethylene }(27 \%) \\
& \text { 11e: } \mathrm{R}=\mathrm{C}_{18} \mathrm{H}_{37}, \mathrm{O}=\text { ethylene }(/) \\
& \text { 11f: } \mathrm{R}=\mathrm{C}_{18} \mathrm{H}_{37}, \mathrm{O}=\text { hexamethylene }(/) \\
& \text { 11g: } \mathrm{R}=\mathrm{Bu}, \mathrm{O}=0 \text {-phenylene }(/) \\
& \text { 11h: } \mathrm{R}=\mathrm{C}_{18} \mathrm{H}_{37}, \mathrm{O}=0 \text {-phenylene }(/)
\end{aligned}
$$

Scheme 2. Synthesis of $N, N^{\prime}$-dialkyldiamines 7 and reductive amination towards bolaamphiphilic sophorolipids 11

The formation of another class of bolaamphiphilic sophorolipids was evidenced in the course of the evaluation of the reductive amination of sophorolipid aldehyde $\mathbf{6}$ with primary amines. When the same reaction conditions were applied as for the reductive amination with secondary amines, a mixture of secondary sophorolipid amines and bolaamphiphilic sophorolipid amines was obtained. The selective formation of $N$-alkyl bolaamphiphilic sophorolipid amines 12 was accomplished by using only 0.5 equivalents of primary amine and applying the same reaction conditions as described for the $N, N^{\prime}$ dialkyl bolaamphiphilic sophorolipids 11 (Scheme 3).
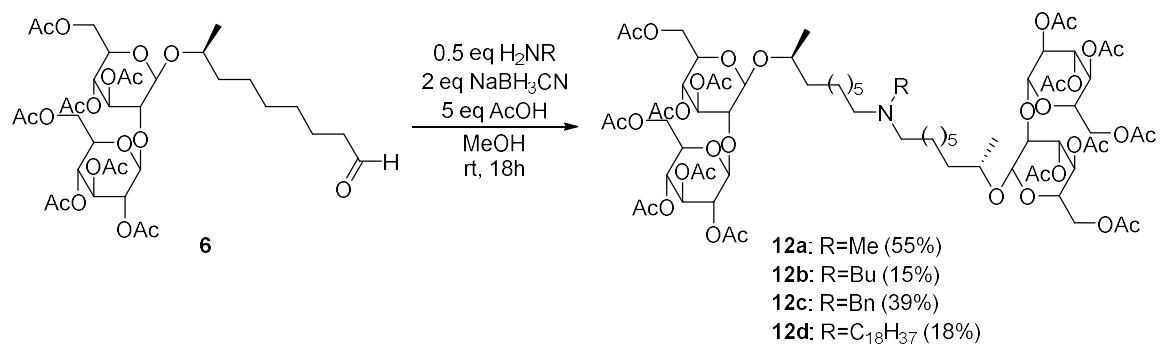

Scheme 3. Reductive amination with primary amines towards bolaamphiphilic sophorolipids $\mathbf{1 2}$

The set of four $N, N^{\prime}$-dialkyl bolaamphiphilic sophorolipids $\mathbf{1 1}$ was transformed into the deprotected $N, N$ '-dialkyl bolaamphiphilic sophorolipids 13, peracetylated dicationic bolaamphiphilic sophorolipids 14 and deprotected dicationic bolaamphiphilic sophorolipids 15 (Scheme 4). The work of De Vos, De Coen et al. showed that the double quaternization of a bis(7-azabicyclo[2.2.1] heptane) derivative with an ethylene linker to a dicationic compound was unsuccessful, even upon reaction with 20 equivalents of methyl iodide at room temperature. ${ }^{45-46}$ Therefore, it could be anticipated that the large charge repulsion on the small ethylene linker could also inhibit the formation of the dicationic bolaamphiphilic sophorolipids 14a and 14c. However, NMR-analysis clearly confirmed the successful synthesis of both compounds. 

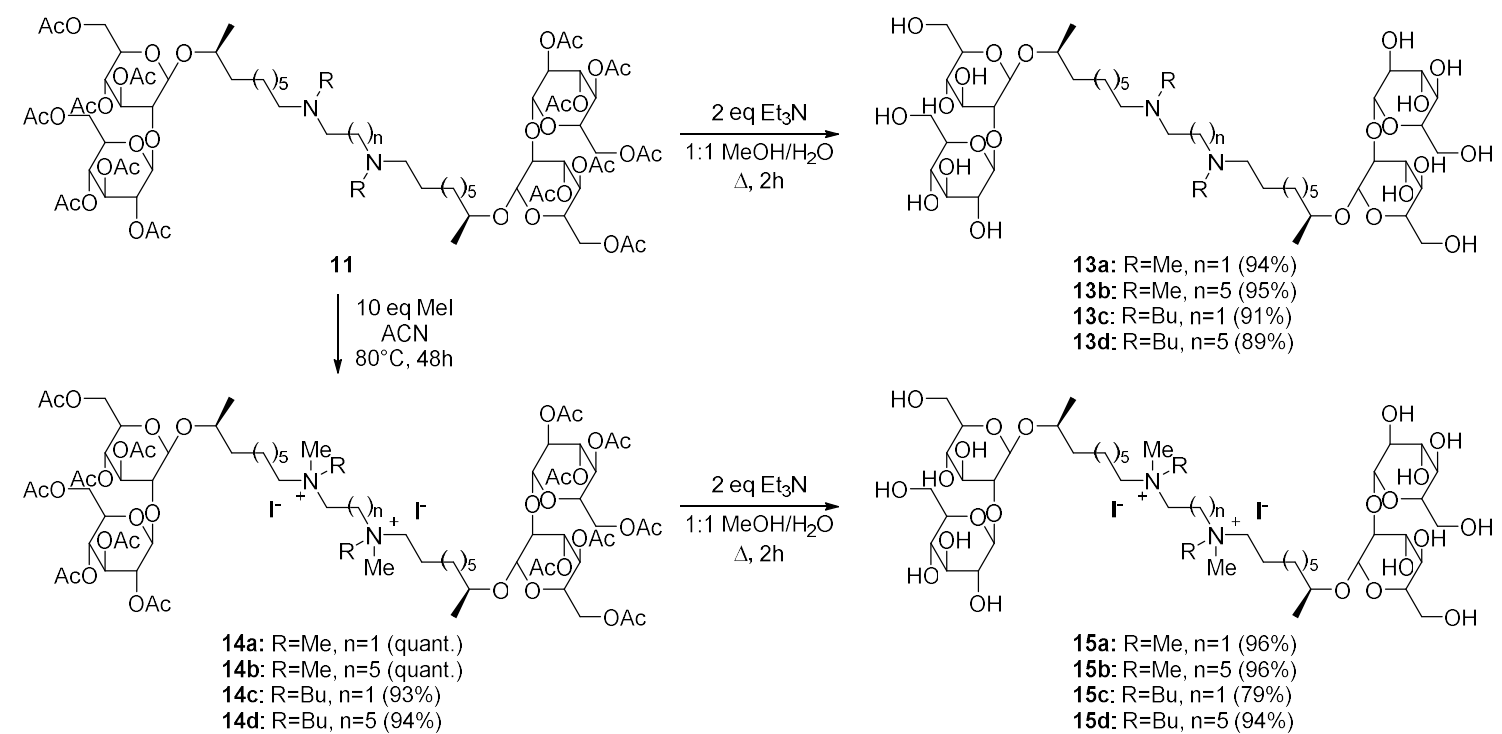

Scheme 4. Modification of $N, N^{\prime}$-dialkyl bolaamphiphilic sophorolipids 11 via quaternization and deprotection

Alternative procedures were evaluated for the synthesis of $N, N^{\prime}$-dialkyl bolaamphiphilic sophorolipids with octadecyl groups on the nitrogen atom. At first, the introduction of the octadecyl groups was attempted via quaternization of the $N, N^{\prime}$-dimethyl bolaamphiphilic sophorolipids 11a and 11b with octadecyl iodide. However, no quaternization occurred in acetonitrile or toluene as solvent after $72 \mathrm{~h}$. An alternative procedure was designed which comprised the formation of secondary bolaamphiphilic sophorolipid amines as intermediates. To prevent similar overalkylations which resulted in the formation of the $N$-alkyl bolaamphiphilic sophorolipid amines 12, the mixture of sophorolipid aldehyde $\mathbf{6}$ and diamine was stirred for $1 \mathrm{~h}$ at room temperature prior to the addition of sodium cyanoborohydride and acetic acid. Attempts to alkylate these secondary bolaamphiphilic sophorolipid amines with octadecyl iodide were unsuccessful. Also reductive amination of these secondary bolaamphiphilic sophorolipid amines with octadecanal failed, although this dual reductive amination procedure proved to work for the synthesis of $N, N$ '-dibutyl bolaamphiphilic sophorolipid 11c. The set of four $N$-alkyl bolaamphiphilic sophorolipids 12 was also transformed into the deprotected $N$-alkyl bolaamphiphilic sophorolipids $\mathbf{1 6}$, peracetylated monocationic bolaamphiphilic sophorolipids 17 and deprotected monocationic bolaamphiphilic sophorolipids 18 (Scheme 5).

To extend the set of $N, N^{\prime}$-dialkyl bolaamphiphilic sophorolipids 11, reductive amination of a $\mathrm{C} 12$ sophorolipid aldehyde $\mathbf{2 0}$ was performed with $N, N^{\prime}$-alkyldiamines 7a, 7b and 7d (Scheme 6). This C12 sophorolipid aldehyde was obtained via incorporation of petroselinic acid into the sophorolipid structure, resulting in an aldehyde intermediate with a longer lipid chain after the ozonolysis reaction. ${ }^{47}$ The resulting $N, N^{\prime}$-dialkyl bolaamphiphilic sophorolipids $\mathbf{1 1 i}, \mathbf{1 1} \mathbf{j}$ and $\mathbf{1 1 k}$ were subsequently quaternized with methyl or butyl iodide towards the peracetylated dicationic bolaamphiphilic sophorolipids 14e-h. In view of the antimicrobial activities for the C9 sophorolipid aldehyde derived bolaamphiphilic compounds (vide infra), only the peracetylated dicationic bolaamphiphilic sophorolipids 14e-h were deprotected towards the dicationic bolaamphiphilic sophorolipids 15e-h. 

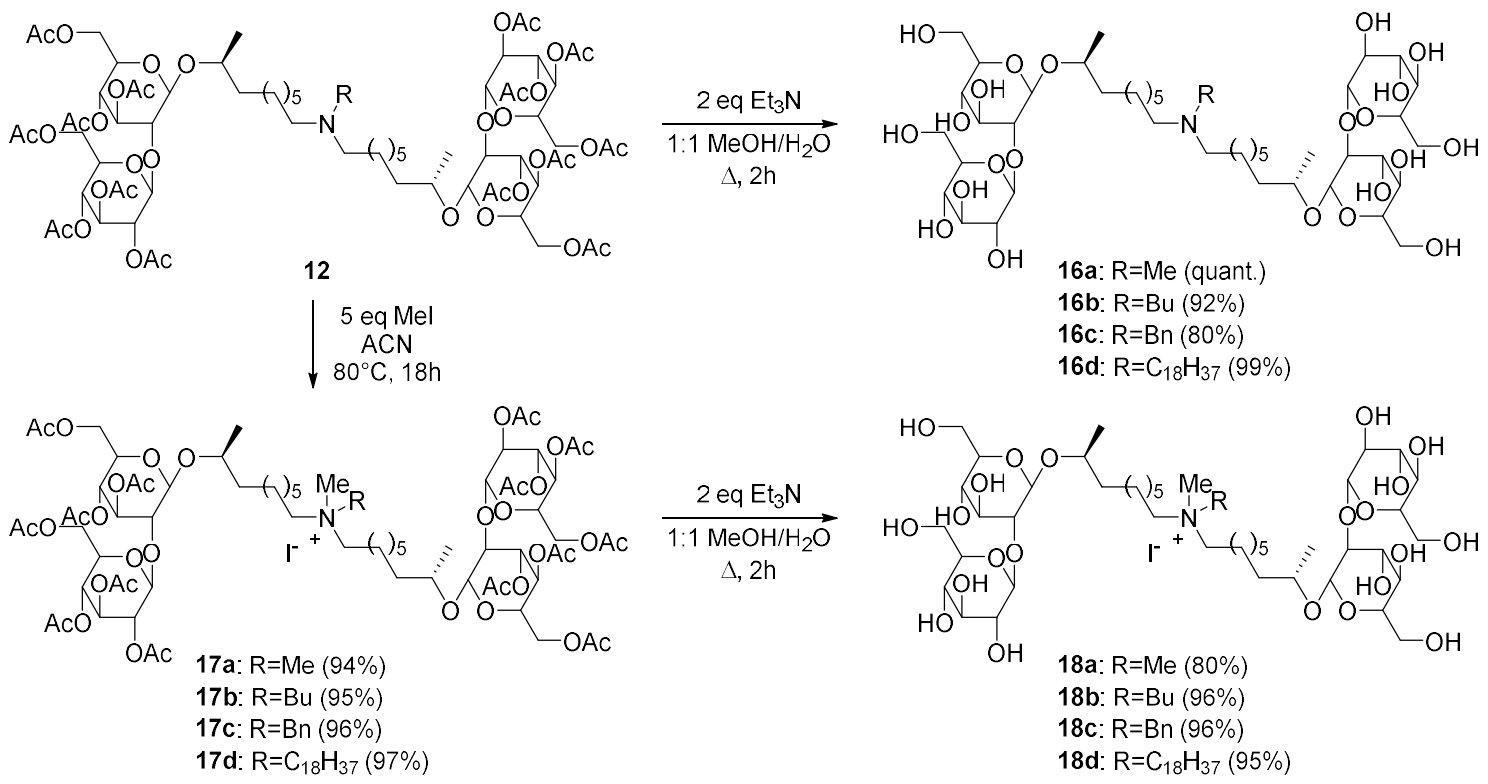

Scheme 5. Modification of $N$-alkyl bolaamphiphilic sophorolipids 12 via quaternization and deprotection

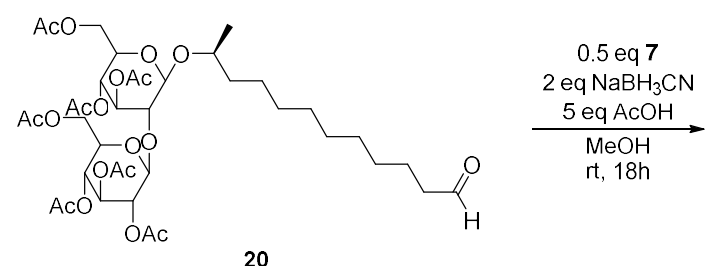

20
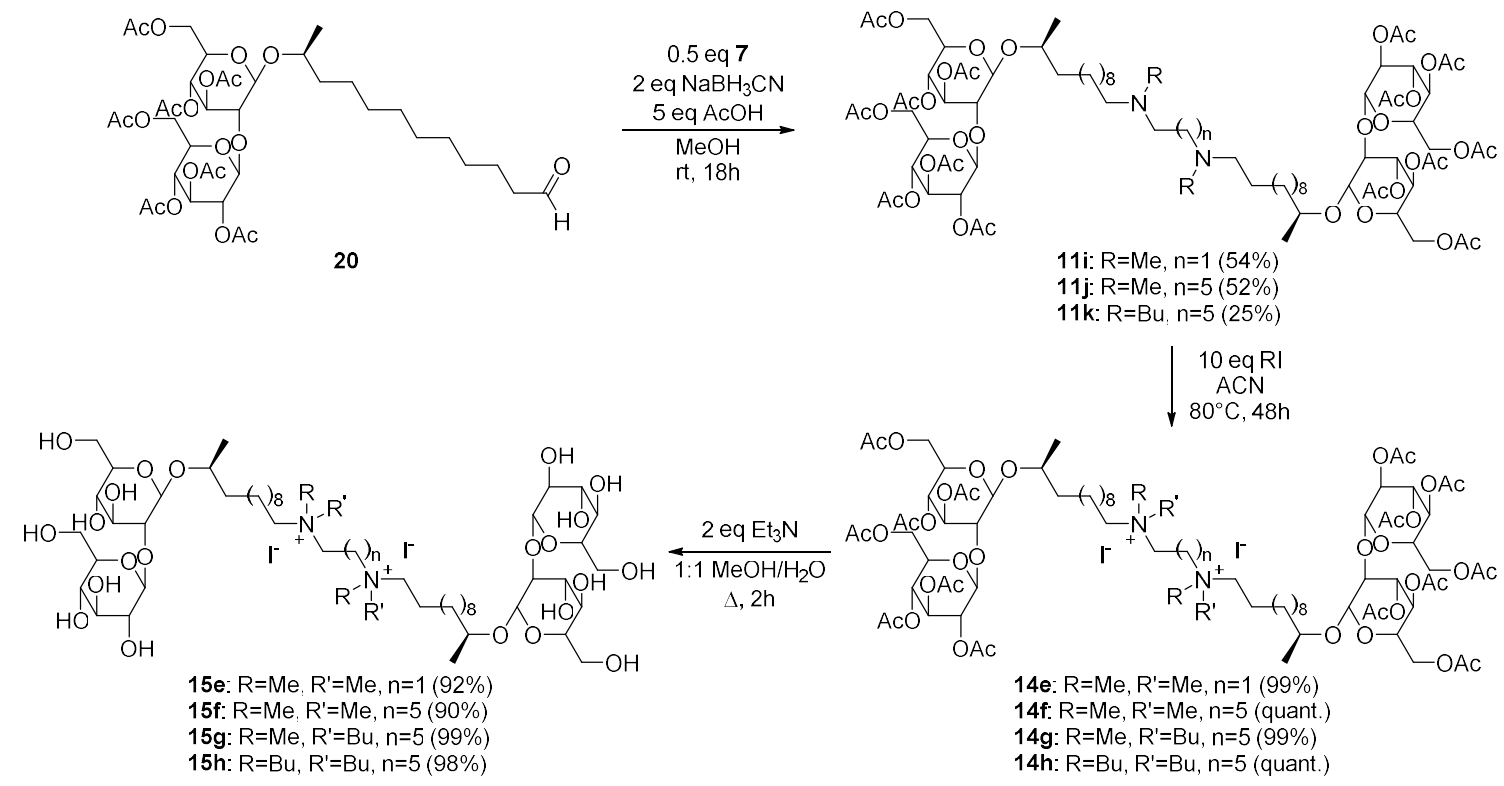

Scheme 6. Modification of $\mathrm{C} 12$ sophorolipid aldehyde 19 towards $N, N^{\prime}$-dialkyl bolaamphiphilic sophorolipipds

Different sustainable chemistry metrics were evaluated for the synthesis of the bolaamphiphilic sophorolipid derivatives (Table 1). Overall reaction yields ranging from 8 to $33 \%$ were obtained for the new derivatives, with the ozonolysis and reductive amination being the crucial steps. ${ }^{37}$ If the yields of these reaction steps could be heightened towards $90 \%$ in an industrial setting by avoiding automated chromatography purification, the overall yield of the reaction pathways could be raised to $71 \%$. For the calculation of the carbon efficiency (CE) and the atom economy (AE), the valorization of the methyl 9oxononanoate byproduct resulting from the ozonolysis step is taken into account. The CE estimates the percentage of carbon from the reactants that is contained in the final product. This metric does not take into account the amount of solvent used and waste generated during the process. The AE estimates the total amount of reactants that are incorporated into the final product. Also here, only the reactants which are incorporated into the final product are taken into account. Solvent use and waste generation are not quantified. Metrics which do take the solvent use and waste generation into account are not qualified 
here since these largely depend on the scale at which the reactions are performed and scale-up of the reaction procedures was out of the scope of this work. For all reaction steps, the use of green solvents was aimed at.

Table 1. Overview of the sustainable chemistry metrics for the production of the bolaamphiphilic sophorolipid derivatives. $\varepsilon=$ reaction yield, $\mathrm{CE}=$ carbon efficiency, $\mathrm{AE}=$ atom economy.

\begin{tabular}{|c|c|c|c|c|c|c|c|}
\hline & $\varepsilon(\%)$ & CE (\%) & AE (\%) & & $\varepsilon(\%)$ & CE (\% & AE (\%) \\
\hline \multicolumn{4}{|c|}{ Peracetvlated $N, N^{\prime}$-dialkvl bolaamphiphilic SL } & \multicolumn{4}{|c|}{ Deprotected $N, N^{\prime}$-dialkvl bolaamphiphilic SL } \\
\hline $11 a$ & 19 & 69 & 60 & 13a & 17 & 44 & 37 \\
\hline 11b & 19 & 70 & 61 & 13b & 18 & 45 & 38 \\
\hline 11c & 24 & 70 & 61 & 13c & 22 & 46 & 38 \\
\hline 11d & 16 & 71 & 62 & 13d & 24 & 48 & 39 \\
\hline $11 \mathbf{i}$ & 32 & 69 & 60 & & & & \\
\hline $\mathbf{1 1 j}$ & 31 & 70 & 61 & & & & \\
\hline $11 \mathrm{k}$ & 15 & 71 & 62 & & & & \\
\hline \multicolumn{4}{|c|}{ Peracetylated $N$-alkyl bolaamphiphilic SL } & \multicolumn{4}{|c|}{ Deprotected $N$-alkyl bolaamphiphilic SL } \\
\hline $12 \mathbf{a}$ & 33 & 69 & 60 & $16 \mathbf{a}$ & 33 & 43 & 37 \\
\hline $12 b$ & 9 & 70 & 61 & 16b & 8 & 44 & 37 \\
\hline $12 \mathrm{c}$ & 23 & 70 & 61 & $16 \mathrm{c}$ & 19 & 45 & 38 \\
\hline $12 d$ & 11 & 72 & 63 & $16 d$ & 11 & 49 & 41 \\
\hline \multicolumn{4}{|c|}{ Peracetylated Dicationic bolaamphiphilic SL } & \multicolumn{4}{|c|}{ Deprotected Dicationic bolaamphiphilic SL } \\
\hline 14a & 18 & 70 & 63 & $15 \mathbf{a}$ & 18 & 44 & 42 \\
\hline 14b & 18 & 70 & 64 & $15 b$ & 18 & 46 & 42 \\
\hline $14 \mathrm{c}$ & 23 & 71 & 64 & $15 c$ & 18 & 47 & 43 \\
\hline 14d & 15 & 72 & 64 & $15 d$ & 14 & 48 & 43 \\
\hline $14 \mathrm{e}$ & 32 & 70 & 63 & $15 \mathrm{e}$ & 29 & 45 & 41 \\
\hline $14 f$ & 31 & 70 & 64 & $15 f$ & 28 & 46 & 42 \\
\hline $14 \mathrm{~g}$ & 15 & 72 & 64 & $15 g$ & 15 & 48 & 43 \\
\hline $14 \mathrm{~h}$ & 15 & 71 & 65 & $15 \mathrm{~h}$ & 14 & 49 & 45 \\
\hline \multicolumn{4}{|c|}{ Peracetylated Monocationc bolaamphiphilic SL } & \multicolumn{4}{|c|}{ Deprotected Monocationc bolaamphiphilic SL } \\
\hline $17 \mathbf{a}$ & 31 & 69 & 62 & $18 \mathbf{a}$ & 25 & 44 & 39 \\
\hline $\mathbf{1 7 b}$ & 9 & 70 & 63 & $18 b$ & 8 & 45 & 40 \\
\hline $17 \mathrm{c}$ & 22 & 71 & 63 & $18 \mathrm{c}$ & 21 & 46 & 40 \\
\hline $17 d$ & 10 & 73 & 65 & 18d & 10 & 49 & 43 \\
\hline
\end{tabular}

\section{Evaluation of the antimicrobial activity}

The antimicrobial activity of the peracetylated and deprotected $N, N^{\prime}$-dialkyl bolaamphiphilic sophorolipids 11 and 13, peracetylated and deprotected dicationic bolaamphiphilic sophorolipids 14 and 15, peracetylated and deprotected $N$-alkyl bolaamphiphilic sophorolipids 12 and 16, and peracetylated and deprotected monocationic bolaamphiphilic sophorolipids 17 and 18 was evaluated. As reference compounds, the previously synthesized quaternary ammonium sophorolipids 19a and 19b with an octadecyl chain on the nitrogen atom were taken into account (Figure 3).
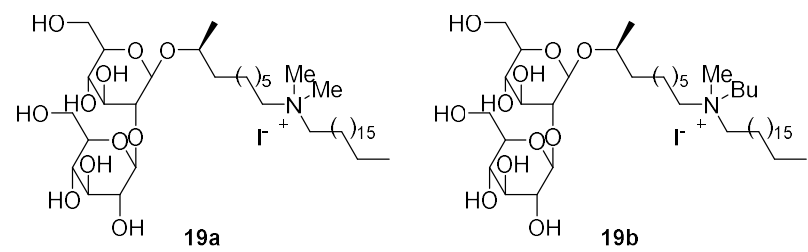

Figure 3. Quaternary ammonium sophorolipids 19a and 19b having demonstrated high transfection efficiencies ${ }^{36}$

The Gram-negative bacteria Escherichia coli LMG 8063, Klebsiella pneumoniae LMG 2095 and Pseudomonas aeruginosa PAO1, and the Gram-positive bacteria Staphylococcus aureus ATCC 6538 and Staphylococcus aureus Mu50 were chosen as test organisms because of their clinical relevance. 
This set includes bacteria of high medical concern, some being part of the WHO priority pathogen list for the development of new antibiotic alternatives. ${ }^{48}$ The bioassay was carried out in 96 -well plates in a concentration series ranging from 2500 to $1.22 \mu \mathrm{g} / \mathrm{mL}$ of test compound and approximately $5 \times 10^{4}$ bacteria in a final volume of $200 \mu \mathrm{L}$. None of the evaluated compounds inhibited growth of any of the three Gram-negative bacteria tested. A set of nine bolaamphiphilic sophorolipids showed activity against one or both $S$. aureus strains. For all nine compounds, both the minimal inhibitory concentration (MIC, i.e. the lowest concentration that inhibits growth) and minimal bactericidal concentration (MBC, i.e. the lowest concentration that kills cells) values were determined against the two $S$. aureus strains (Table S1 ESI). All activities were determined in duplicate or triplicate. Results are considered reproducible when the repetitions don't differ more than one dilution. In case a higher difference is obtained, the highest value is reported.

Within this set of bolaamphiphilic sophorolipid derivatives, the peracetylated dicationic and monocationic congeners $\mathbf{1 4}$ and $\mathbf{1 7}$ proved to be the most active groups of derivatives. The highest activity was obtained for peracetylated $N, N^{\prime}$-dibutyl- $N, N^{\prime}$-dimethylethylene bolaamphiphilic sophorolipid diammonium di-iodide 14c with MIC and MBC values against $S$. aureus ATCC 6538 of 39 and $312 \mu \mathrm{g} / \mathrm{mL}(=20$ and $158 \mu \mathrm{M})$, respectively, and MIC and MBC values against $S$. aureus Mu50 of 156 and $312 \mu \mathrm{g} / \mathrm{mL}$ (=79 and $158 \mu \mathrm{M}$ ), respectively. For a better comparison of the active bolaamphiphilic derivatives with each other and with the previously synthesized quaternary ammonium sophorolipids, the MIC and MBC values were converted based on their molecular weight (Table 2). On this basis, $N, N$ '-dibutyl- $N, N$ '-dimethylethylene bolaamphiphilic sophorolipid diammonium diodide $\mathbf{1 4 c}$ still has the highest activity among all bolaamphiphilic derivatives. However, these MIC values are still fourfold higher than the ones obtained for the deprotected quaternary ammonium sophorolipids $\mathbf{1 9}$ with an octadecyl chain on the nitrogen atom.

Table 2. MIC and MBC values $(\mu \mathrm{M})$ for the active compounds against S. aureus ATCC 6538 and S. aureus Mu50. The best results for the new derivatives are highlighted in bold. For comparison, the results

\begin{tabular}{lcccccccccccc}
\hline$(\boldsymbol{\mu M})$ & & $\mathbf{1 1 b}$ & $\mathbf{1 4 a}$ & $\mathbf{1 4 b}$ & $\mathbf{1 4 c}$ & $\mathbf{1 4 d}$ & $\mathbf{1 7 a}$ & $\mathbf{1 7 b}$ & $\mathbf{1 7 c}$ & $\mathbf{1 8 d}$ & $\mathbf{1 9 a}$ & $\mathbf{1 9 b}$ \\
\hline S. aureus & MIC & 375 & 165 & 80 & 20 & 38 & 369 & 360 & 353 & 116 & 2.18 & 2.09 \\
ATCC 6538 & MBC & $>1501$ & 330 & 321 & 158 & 153 & 1476 & 720 & $>1412$ & 233 & 8.76 & 2.09 \\
\hline S. aureus & MIC & 1501 & 660 & 321 & 79 & 153 & 185 & 360 & 176 & 233 & 4.37 & 4.18 \\
Mu50 & MBC & 1501 & 1320 & 321 & 158 & 614 & 1476 & 1440 & 706 & $>1861$ & 17.5 & 16.7 \\
\hline
\end{tabular}

Antimicrobial activities were also evaluated for the petroselinic acid based peracetylated and deprotected dicationic bolaamphiphilic sophorolipids (Table 3, Table S2 ESI). Low activities were observed against the Gram-negative bacteria, but five of the derivatives showed moderate to high activity against the Gram-positive bacteria. The highest activity was obtained for the peracetylated $N, N^{\prime}-$ dibutyl,- $N, N^{\prime}$-dimethylhexamethylene bolaamphiphilic sophorolipid diammonium di-iodide $\mathbf{1 4 g}$ and $N, N, N$ ', $N$ '-tetrabutylhexamethylene bolaamphiphilic sophorolipid diammonium di-iodide $\mathbf{1 4 h}$, which are almost in the same range as for the previously synthesized deprotected quaternary ammonium sophorolipids with an octadecyl chain on the nitrogen atom. The natural sophorolipid lactone and acid were also evaluated in the antimicrobial analysis. The activity for oleic acid based sophorolipid acid 2 and lactone 3 are displayed in Table 3. These results illustrate that the chemical modification enables an increase in the antimicrobial activity by a factor 100 .

In the evaluation, an S. aureus Mu50 strain is included, which is a methicillin-resistant Staphylococcus aureus (MRSA) strain with vancomycin resistance. MIC values for activity of vancomycin and clindamycin against this $S$. aureus Mu50 were reported to be $8 \mu \mathrm{g} / \mathrm{mL}(=6 \mu \mathrm{M})$ and $512 \mu \mathrm{g} / \mathrm{mL}(=1205$ $\mu \mathrm{M}$ ), respectively. ${ }^{49}$ Therefore, the activities obtained with compounds $14 \mathrm{~g}$ and $\mathbf{1 4 h}$ against both $S$. aureus strains are reasonably good. However, it should be taken into account that these results are obtained with in vitro testing and that further in vivo testing is necessary to determine the actual antibiotic 
potential of these compounds. In a next step, toxicity on eukaryotic cells should be evaluated to know whether the antimicrobial activity occurs at a lower concentration.

Table 3. MIC and MBC values $(\mu \mathrm{M})$ for the active compounds against E. coli LMG 8063, K. pneumoniae LMG 2095, S. aureus ATCC 6538 and $S$. aureus Mu50. ND = not determined.

\begin{tabular}{lcccccccccc}
\hline ( $\boldsymbol{\mu M})$ & & $\mathbf{2}$ & $\mathbf{3}$ & $\mathbf{1 4 e}$ & $\mathbf{1 4 f}$ & $\mathbf{1 4 g}$ & $\mathbf{1 4 h}$ & $\mathbf{1 5 h}$ & $\mathbf{1 9 a}$ & $\mathbf{1 9 b}$ \\
\hline E. coli & MIC & $>1607$ & $>1453$ & $>506$ & 492 & 472 & 454 & $>620$ & $>1122$ & $>1071$ \\
LMG 8063 & MBC & ND & ND & ND & ND & ND & ND & ND & ND & ND \\
\hline K. pneumoniae & MIC & $>1607$ & $>1453$ & 253 & 246 & 236 & 227 & $>620$ & $>1122$ & $>1017$ \\
LMG 2095 & MBC & ND & ND & ND & ND & ND & ND & ND & ND & ND \\
\hline S. aureus & MIC & $>1607$ & 182 & 63.2 & 15.3 & 3.69 & 3.55 & 620 & 2.18 & 2.09 \\
ATCC 6538 & MBC & ND & 363 & $>506$ & 30.7 & 7.37 & 3.55 & ND & 8.76 & 2.09 \\
\hline S. aureus & MIC & $>1607$ & 363 & 63.2 & 15.3 & 7.37 & 7.09 & 310 & 4.37 & 4.18 \\
Mu50 & MBC & ND & 1453 & $>506$ & 246 & 14.8 & 7.09 & ND & 17.5 & 16.7 \\
\hline
\end{tabular}

\section{Evaluation of the transfection efficiency}

Transfection efficiencies for the bolaamphiphilic sophorolipids were evaluated in a similar way as for the previously synthesized quaternary ammonium sophorolipids. ${ }^{36}$ High transfection results were obtained for the two derivatives having an octadecyl chain on the nitrogen atom (Figure 3). Lipofectamine 3000 (LFM), a commercial lipofection reagent, was used as a reference compound in the transfection experiments. The application of quaternary ammonium derivatives for the compaction and delivery of plasmid DNA (pDNA) and nucleic acids to different cell lines, both in vitro and in vivo, is already widely known. ${ }^{50-54}$ Renewable based gene delivery vectors are of special interest since they are expected to have an increased biocompatibility. ${ }^{40,55-57}$

From the set of thirty-two bolaamphiphilic sophorolipids, three cationic compounds (15b, 15d and $\mathbf{1 8 d})$ were evaluated for their ability to form supramolecular aggregates in water solution by using the lipid film hydration method. For all liposomal solutions, the size of the particles and their surface charge were determined via Dynamic Light Scattering (DLS) and zeta measurements, respectively. The formation of a homogeneous formulation is a necessary prerequisite for the evaluation of the suitability of quaternary ammonium salts as vectors for gene delivery. The compounds were formulated with or without 1,2-dioleyl-sn-glycero-3-phosphoethanolamine (DOPE). Formulation with DOPE proved to be necessary to obtain homogenous formulations (Table 4), as was previously reported for similar compounds. ${ }^{36}$ For the monocationic bolaamphiphilic sophorolipid 18d, a positive zeta potential was obtained as is expected for liposomes generated from cationic lipid derivatives. However, for both dicationic bolaamphiphilic sophorolipids $\mathbf{1 5 b}$ and $\mathbf{1 5 d}$, negative zeta potentials were obtained for formulations both with and without DOPE. Therefore, only compound 18d was considered for further evaluation of the transfection efficiency.

Table 4. Size and zeta potential measurements of liposomal solutions prepared at $1.5 \mathrm{mM}$ after 2 days of hydration without (left) or with (right) DOPE. ND = not determined.

\begin{tabular}{ccccccc}
\hline & Size (nm) & Polydisp. index & Zeta $(\mathbf{m V})$ & Size (nm) & Polydisp. index & Zeta (mV) \\
\cline { 5 - 6 } & & & & & + DOPE & \\
\hline 15b & ND & ND & ND & $432 \pm 15$ & 0.41 & -20.1 \\
15d & $73 \pm 11$ & 1 & -0.39 & $175 \pm 0.1$ & 0.13 & -24.1 \\
18d & ND & ND & ND & $103 \pm 1$ & 0.28 & 44 \\
\hline
\end{tabular}

The capacity to compact pDNA was evaluated for the liposomal formulation of compound 18d with DOPE by pDNA retardation assays on agarose gel electrophoresis (Figure 4). This was performed at different charge ratios $(\mathrm{CR})$ in a similar way as for the previously synthesized quaternary ammonium sophorolipids 19a-b. CR is defined as the number of positive charges provided by the cationic lipid 
derivative divided by the number of negative charges carried by the pDNA. The previously synthesized quaternary ammonium sophorolipids 19a-b show a low capacity to complex pDNA at each CR. A similar behavior was observed for compound $\mathbf{1 8 d}$.

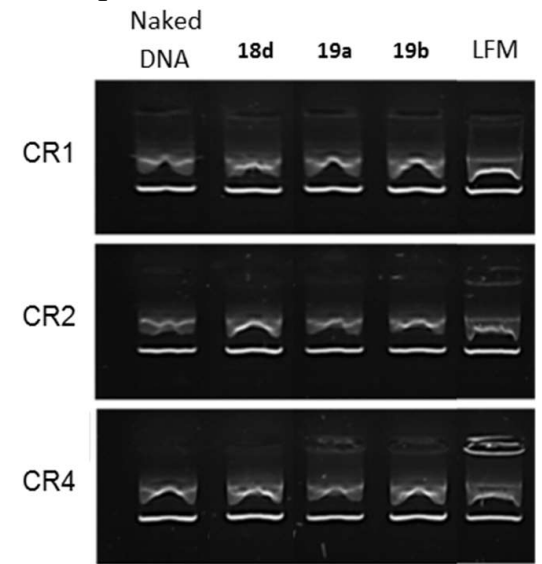

Figure 4. DNA complexation ability of compound 18d (formulated with DOPE) as determined via the agarose gel retardation assay for different charge ratios. Previously synthesized quaternary ammonium sophorolipids 19a and 19b, naked DNA and LFM (Lipofectamine 3000) were used as controls.

In the transfection assay, compound $\mathbf{1 8 d}$ was evaluated considering its ability to deliver DNA to three human-derived cell lines, namely melanoma cells (SKMEL28) and two airway epithelial cells i.e. (i) lung carcinoma (A549) and (ii) normal bronchial (16HBE) cells. A reporter (luciferase-encoding) pDNA was used that allowed the determination of the transfection efficiency via highly sensitive luminescence measurements. The formulation of compound 18d with DOPE was evaluated at different CR. Compared with the previously synthesized quaternary ammonium sophorolipids 19a-b and the control LFM, compound 18d was almost ineffective for the transfection of the three cell lines (Figure 5). No real toxicity was observed towards any cell line (Figure 6).

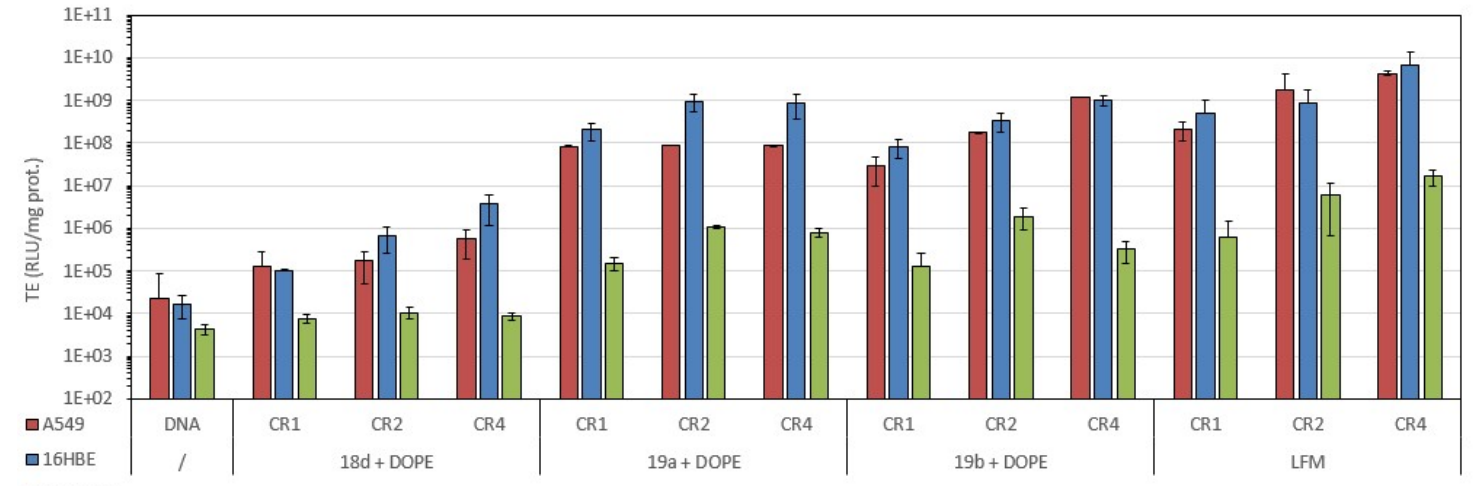

Figure 5. Transfection efficacies (TE) of compound 18d formulated with DOPE on three cell lines (A549, 16HBE and SKMEL28) using luciferase-encoding pDNA (DNA). TE are expressed in RLU per mg of proteins $(\mathrm{n}=3)$. Lipofectamine (LFM) and naked (uncomplexed) pDNA were used as positive and negative controls, respectively. 


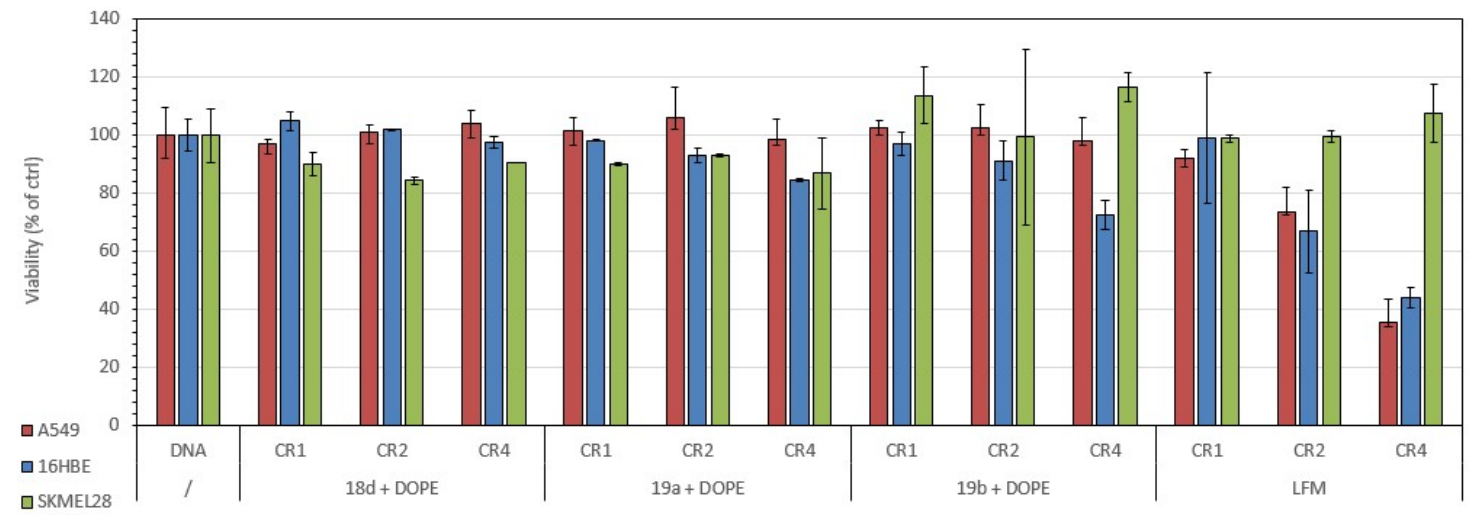

Figure 6. Cell viability determined $48 \mathrm{~h}$ after incubation of the cells with lipoplexes prepared with compound 18d formulated with DOPE. Naked pDNA (DNA) was used as the negative control. Values are expressed as a percentage of the viability determined with untransfected cells.

\section{Evaluation of the self-assembly properties}

In an attempt to correlate the antimicrobial and transfection data, the self-assembly behavior in water of the bolaamphiphilic sophorolipids was evaluated via Small-Angle X-ray Scattering (SAXS) analysis. All samples were analyzed in milli-Q grade water within $24 \mathrm{~h}$ after sample preparation in a broad concentration range $(0.78-100 \mathrm{mg} / \mathrm{mL})$. In the following discussion, we only report the SAXS data at $6.25 \mathrm{mg} / \mathrm{mL}(=3.88-4.79 \mathrm{mM})$ for the dicationic bolaamphiphilic sophorolipids 15 , at $10 \mathrm{mg} / \mathrm{mL}(=8.32-$ $10.38 \mathrm{mM}$ ) for the $N$-alkyl bolaamphiphilic sophorolipids 16 and at $12.5 \mathrm{mg} / \mathrm{mL}(=9.30-11.31 \mathrm{mM})$ for the monocationic bolaamphiphilic sophorolipids 18. The choice to show this set of concentration values is a good compromise between a scattering signal with a satisfactory signal-to-noise ratio and the range of concentrations used in the previous experiments. Figures 7-9 show the SAXS data of only deprotected dicationic bolaamphiphilic sophorolipids 15a-15g, $N$-alkyl bolaamphiphilic sophorolipids 16a-16d and monocationic sophorolipids 18a-18d, because the peracetylated derivatives are poorly soluble in water. According to the SAXS data, the self-assembly properties of the quaternary ammonium bolaamphihilic sophorolipids are neither uniform nor straightforward to interpret. The group of compounds $\mathbf{1 5}$ (a, e-h) shows a poor, although clearly present, scattering signal. A qualitative analysis of the data using a Guinier approach provides a set of radii of gyration $\left(\mathrm{R}_{\mathrm{g}}\right)$ below $1 \mathrm{~nm}$ (Table 4$) .{ }^{58}$ By using the classical Tanford formula

$$
1_{\mathrm{c}}(\AA)=1.5+1.265 \mathrm{n}_{\mathrm{c}} \quad(1)
$$

(with $1_{c}$ and $n_{c}$ being the hydrocarbon chain length and the number of carbon atoms in the chain respectively) to estimate the length of the fully extended hydrocarbon chain, one can estimate the approximate overall size of any of these compounds to range between 5 and $6 \mathrm{~nm}$. ${ }^{59}$ Under these circumstances, the aggregates probed by SAXS must be composed of few, folded, bolaform compounds 15 (a,e-h). The set of compounds 15b-d, of which the $\mathrm{R}_{\mathrm{g}}$ is difficult to estimate, seems to be composed of larger self-assembled objects of undefined morphology. The value of the slope in the $\log (\mathrm{I})-\log (\mathrm{q})$ profiles can help, in some cases, identifying the qualitative nature of the morphology, or the fractional dimension. ${ }^{58}$ In the present case, compounds $\mathbf{1 5 c}$ and $\mathbf{1 5 d}$ have slopes close to -1 (Table 4 ), which could indicate the presence of elongated, cylindrical objects although compound 15d has a more complex profile. The value of the slope found for compound $\mathbf{1 5 b}(-1.79 \pm 0.03)$ and its poor overall scattering signal do not allow a precise interpretation although values between -1 and -2 have been reported for flexible cylinders. 

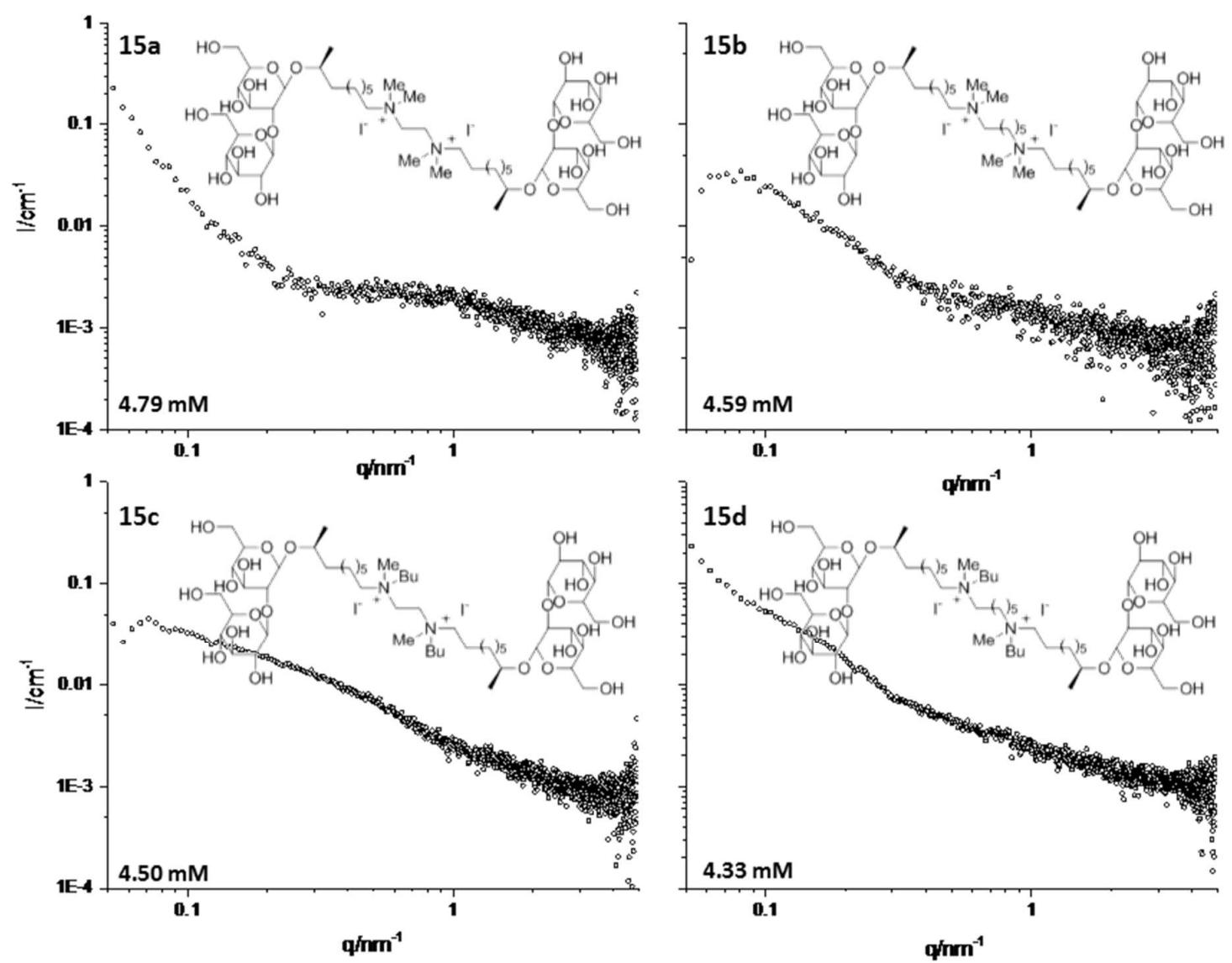

Figure 7. SAXS data of oleic acid based dicationic bolaamphiphilic sophorolipids 15a-15d at a concentration of $6.25 \mathrm{mg} / \mathrm{mL}$ $(=4.33-4.79 \mathrm{mM})$.

In the family of compounds $\mathbf{1 6}$ and $\mathbf{1 8}$, one can find strong similarities in the self-assembly behavior. Compounds 16a, 16b, 18a and $18 \mathrm{~b}$ are all characterized by a scattering profile providing an estimated $\mathrm{R}_{\mathrm{g}}<1$ and, above all, an important low-q scattering signal indicating the presence of larger objects. For compounds $\mathbf{1 8 a}$ and $\mathbf{1 8 b}$, the slopes can be evaluated in the vicinity of -1.6 (Table 5), which is a value observed for elongated wormlike micelles. ${ }^{28,60-62}$ Compounds 16c and 18c have typical scattering profiles of crystalline flat fibers, characterized by a -2 slope in the low-q range and a diffraction peak, here localized at $\mathrm{q}=2.28 \mathrm{~nm}^{-1}$, and indicative of a repeating distance between the bolaform lipid planes of $2 \pi / \mathrm{q}=2.75 \mathrm{~nm}$. Similar data have been reported for several sophorolipid-based systems forming twisted nanoscale fibers. ${ }^{31,63}$ Finally, compounds $\mathbf{1 6 d}$ and $\mathbf{1 8 d}$ have the typical profile of spheroidal micelles in the presence of repulsive interactions, which can be recognized by the flattened low-q signal for 16d and the broad interaction peak for 18d. Similar data have been reported for acidic sophorolipids and acidic sophorolipids mixed with sodium dodecyl sulfate..$^{30,64}$

As a general comment, irrespective of the mono- and diammonium linker in the bolaform sophorolipids, the nature of the substitute strongly impacts the self-assembly behavior of these molecules. In the case of methyl or butyl groups (compounds 15a-h, 16a-b and 18a-b), the self-assembly properties are rather poor. In some cases, small molecular aggregates are formed in the presence of larger objects of which the morphology is rather unclear without further microscopy studies, which are out of the scope of this work. On the contrary, the octadecyl hydrocarbon group on samples 16d and 18d drives the selfassembly of the bolaform sophorolipids into micelles, most likely through the hydrophobic interaction which is a well-known fact in the theory of the self-assembly of amphiphiles. ${ }^{59,65-66}$ Similar results have been observed on several long-chained quaternary ammonium sophorolipids such as compounds 19a 
and 19b (unpublished results). Intermicellar repulsive interactions characterize these systems and the strength of the interaction depends on the charge localized on the nitrogen. An ammonium group (18d) favors electrostatic interactions (well-defined, broad scattering peak) which were previously described also for sophorolipid systems, while an amino group favors less pronounced, excluded volume, interactions (flattened low-q signal). ${ }^{30,64}$ Finally, the presence of an aryl group favors $\pi$ - $\pi$ stacking which is known to drive low molecular weight molecules, including bolaform glycolipids, towards the formation of self-assembled fibers. ${ }^{67}$

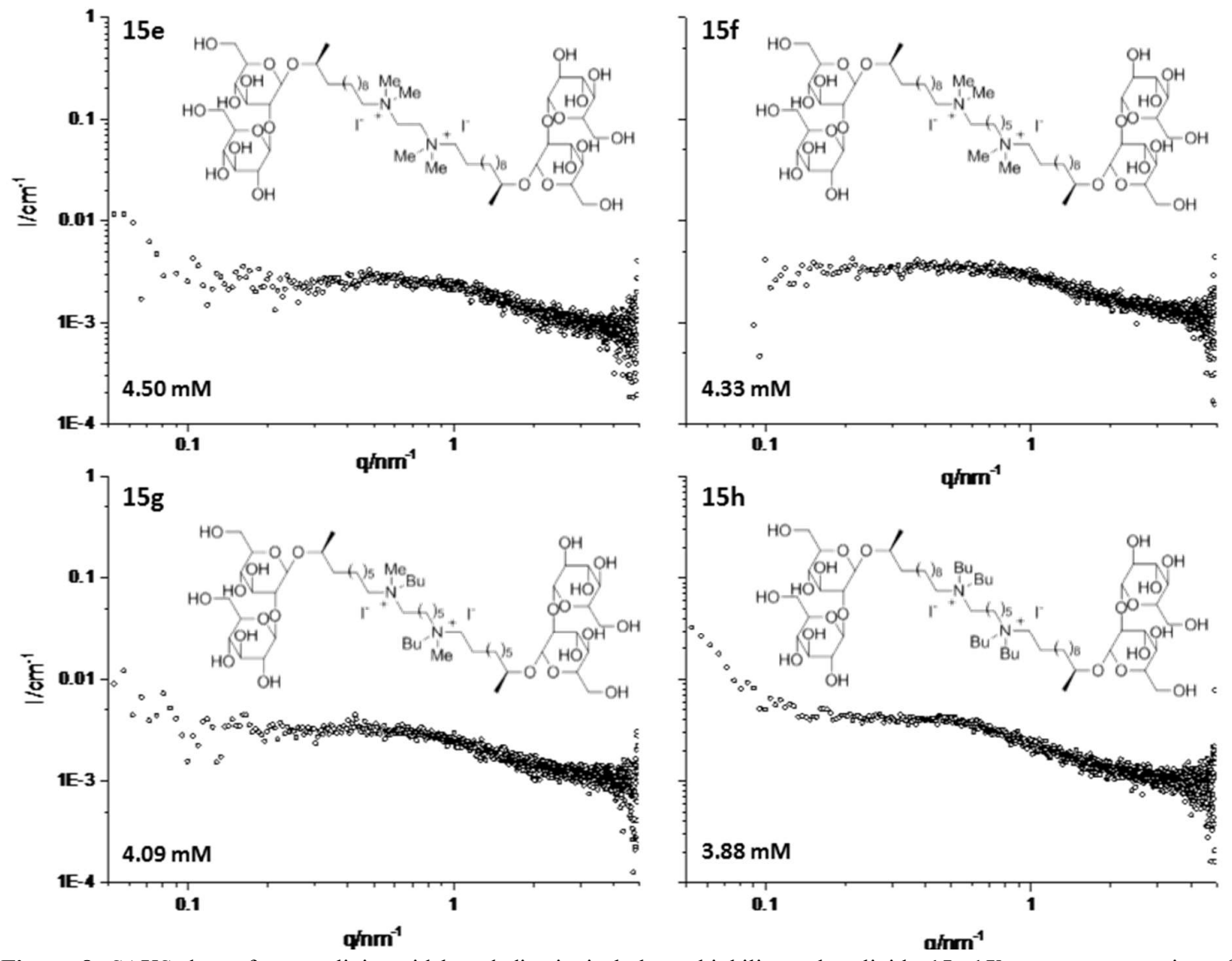

Figure 8. SAXS data of petroselinic acid based dicationic bolaamphiphilic sophorolipids $\mathbf{1 5 e - 1 5 h}$ at a concentration of $6.25 \mathrm{mg} / \mathrm{mL}(=3.88-4.50 \mathrm{mM})$.

Among all non-acetylated molecules, monocationic bolaamphiphilic sophorolipid 18d, having an octadecyl substituent, was the only compound which proved to be interesting for the evaluation of the transfection properties. However, this compound was much less efficient in comparison to the previously synthesized compounds $\mathbf{1 9} \mathbf{a}-\mathbf{b}$, and the reference lipofection reagent Lipofectamine. Interestingly, 18d was the only non-acetylated bolaamphiphilic compound for which antimicrobial activity could actually be observed. Also here, the activity was much lower compared to the previously synthesized compounds 19a-b. Recent work on several long-chained quaternary ammonium sophorolipids, such as compounds $19 \mathbf{a}$ and $\mathbf{1 9 b}$, demonstrates a strong correlation between the antimicrobial or transfection efficiencies and the self-assembly properties of the compounds (submitted results). The explanation is not straightforward for the relationship between self-assembly and transfection efficiency. Different experimental conditions were applied for the measurement of the transfection and self-assembly properties. Self-assembly properties were evaluated with compounds independently solubilized in water, whereas sophorolipids are associated with DOPE (1:1 ratio) to form liposomes that are subsequently mixed with pDNA for the transfection experiments. In consequence, the SAXS results are worth only for the sophorolipid derivatives placed in water but cannot be extended 
to the liposomal solutions where DOPE likely plays an important role for the supramolecular structuration. Mixtures with DOPE are not included in the self-assembly analysis because of the overlap between the signal of micelles and liposomes in the $0.8 \mathrm{~nm}^{-1}<\mathrm{q}<-3 \mathrm{~nm}^{-1}$ region. In the current case, self-assembly confirms that compound $\mathbf{1 8 d}$ is better suited for transfection and antimicrobial action with respect to the other non-acetylated compounds. However, if micelle formations seems to be a necessary condition for one of the above-tested non-acetylated compounds to display antimicrobial and transfection properties, it is definitely not sufficient to guarantee a high performance, which can depend on the interaction between the compound and DOPE, for transfection, and between the molecule and the bacterial strain, for the antimicrobial activity.

Table 5. Radii of gyration, $R_{g}$, low-q slopes and position of diffraction peaks obtained from the SAXS data presented in Figure 7, Figure 8 and Figure 9. Values of $\mathrm{R}_{\mathrm{g}}$ have been determined using the Guinier treatment.

\begin{tabular}{cccc}
\hline & Rg $(\mathbf{n m})$ & Slope & Peak $\left(\mathbf{n m}^{-1}\right)$ \\
\hline $\mathbf{1 5 a}$ & 0.67 & $-3.12 \pm 0.09$ \\
$\mathbf{1 5 b}$ & & $-1.79 \pm 0.03$ & \\
$\mathbf{1 5 c}$ & & $-0.94 \pm 0.01$ & \\
$\mathbf{1 5 d}$ & & $-0.82 \pm 0.03(\mathrm{q}>0.3)$ & \\
$\mathbf{1 5 e}$ & 0.67 & $-1.40 \pm 0.01(\mathrm{q}<0.15)$ & \\
$\mathbf{1 5 f}$ & 0.66 & \\
$\mathbf{1 5 g}$ & 0.69 & \\
$\mathbf{1 5 h}$ & 0.82 & \\
$\mathbf{1 6 a}$ & 0.67 & \\
$\mathbf{1 6 b}$ & 0.51 & & \\
$\mathbf{1 6 c}$ & & & \\
$\mathbf{1 6 d}$ & 3.80 & & \\
$\mathbf{1 8 a}$ & 0.74 & $-1.67 \pm 0.01(\mathrm{q}<0.20)$ & \\
$\mathbf{1 8 b}$ & 0.75 & $-1.72 \pm 0.02(\mathrm{q}<0.20)$ & \\
$\mathbf{1 8 c}$ & & $-2.04 \pm 0.02$ & 2.28 \\
$\mathbf{1 8 d}$ & 3.20 & & \\
\hline
\end{tabular}




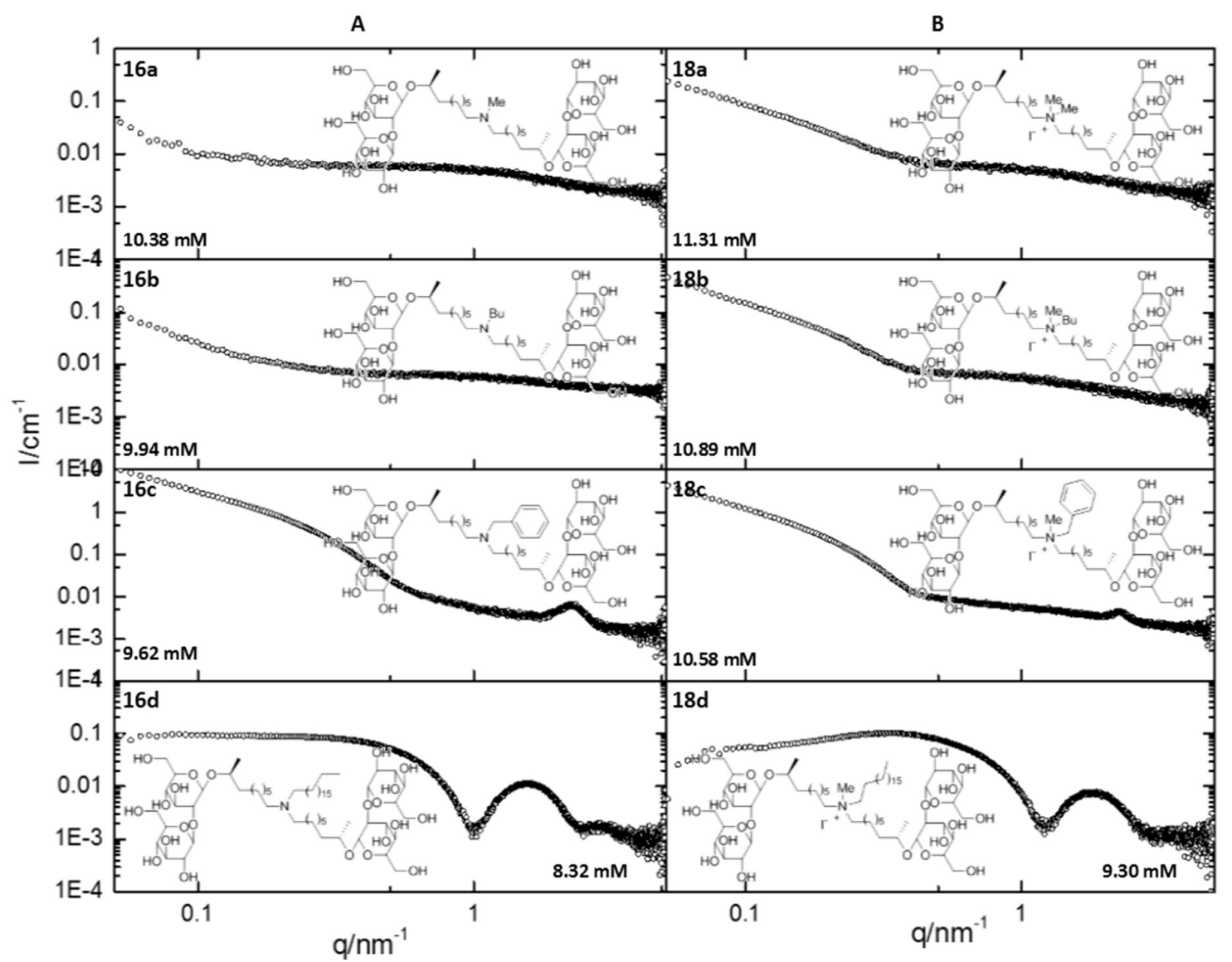

Figure 9. A) SAXS data of $N$-alkyl bolaamphiphilic sophorolipids 16a-16d at a concentration of $10 \mathrm{mg} / \mathrm{mL}(=8.32-10.38 \mathrm{mM})$ and $\mathrm{pH}$ 7. B) SAXS data of monocationic bolaamphiphilic sophorolipids $18 \mathrm{a}-\mathbf{1 8 d}$ at a concentration of $12.5 \mathrm{mg} / \mathrm{mL}(=9.30-$ $11.31 \mathrm{mM})$.

\section{Conclusions}

A new set of sophorolipid derivatives, i.e. bolaamphiphilic sophorolipids, was synthesized starting from a sophorolipid aldehyde intermediate via reductive amination with diamines or primary amines. Attempts to introduce octadecyl substituents or an aromatic linker on the diamine bolaamphiphilic sophorolipids were unsuccessful, probably due to the poor solubility of the $N, N$ '-octadecyldiamines and steric hindrance induced by the aromatic linker. The new bolaamphiphilic sophorolipids were evaluated for their antimicrobial, transfection and self-assembly properties. The antimicrobial activity was evaluated against the Gram-positive bacteria $S$. aureus ATCC 6538 and S. aureus Mu50, and against the Gram-negative bacteria E. coli LMG 8063, K. pneumoniae LMG 2095 and P. aeruginosa PAO1. Fourteen of the derivatives were active against the Gram-positive bacteria tested, while five of them also showed low activity against the Gram-negative bacteria. The peracetylated dicationic and monocationic congeners 14 and 17 proved to be the most active groups of derivatives. The best results were obtained for the peracetylated $N, N^{\prime}$-dibutyl- $N, N^{\prime}$-dimethylhexamethylene bolaamphiphilic sophorolipid diammonium di-iodide $\mathbf{1 4 g}$ and $N, N, N^{\prime}, N^{\prime}$-tetrabutylhexamethylene bolaamphiphilic sophorolipid diammonium di-iodide $\mathbf{1 4 h}$, with activities almost in the same range as for the previously synthesized deprotected quaternary ammonium sophorolipids with an octadecyl chain on the nitrogen atom. Transfection efficiencies were evaluated for three cationic bolaamphiphilic sophorolipids, namely $\mathbf{1 5 b}$, $15 \mathrm{c}$ an 18d. Formulation with DOPE was required to obtain homogeneous liposomal solutions and a positive zeta potential (as expected for liposomes with cationic lipid derivatives) was only obtained for the $N$-octadecyl bolaamphiphilic sophorolipid ammonium iodide 18d. Then, the ability of the liposomal 
formulation with compound $\mathbf{1 8 d}$ to compact pDNA was assessed. A low compaction was observed for compound 18d, as was also the case for the previously synthesized quaternary ammonium sophorolipids. The capacity of compound 18d to achieve transfection of eukaryotic cells was assessed on three cell lines (A549, 16HBE and SKMEL28). Compared with the previously synthesized quaternary ammonium sophorolipids and the control LFM, compound 18d was almost inefficient for the transfection of the three cell lines and no real toxicity was observed towards any of these cell lines. Evaluation of the selfassembly properties indicated that the presence of an octadecyl chain was necessary for the compounds to self-assemble as micelles. The molecular self-assembly and the net charge of the compounds seem to have an influence on the antimicrobial activity and transfection efficiency. These results are promising to further extend the existing set of bolaamphiphilic sophorolipids and their use for medical and selfassembly applications.

\section{Acknowledgements}

The research leading to these results has received funding from the Long Term Structural Methusalem funding by the Flemish Government (grant number BOF09/01M00409). This work received financial support by the European Synchrotron Radiation Facility (ESRF), Grenoble, France, under the experiment number MX1821. This work benefited from the use of the SasView application, originally developed under NSF award DMR-0520547. SasView contains code developed with funding from the European Union's Horizon 2020 research and innovation programme under the SINE2020 project, grant agreement No 654000 .

\section{Supporting information}

An experimental section on the general instrumental methods and general synthesis procedures is included in the supporting information, together with two tables on the antimicrobial activities of the compounds $(\mu \mathrm{g} / \mathrm{mL})$, the characterization of the synthesized compounds and the accompanying ${ }^{1} \mathrm{H}$ - and ${ }^{13} \mathrm{C}-\mathrm{NMR}$ spectra.

\section{Notes and references}

1. Fuhrhop, A. H.; Wang, T. Y., Bolaamphiphiles. Chem Rev 2004, 104 (6), 2901-2937, DOI $10.1021 / \mathrm{cr} 030602 \mathrm{~b}$.

2. Benvegnu, T.; Brard, M.; Plusquellec, D., Archaeabacteria bipolar lipid analogues: structure, synthesis and lyotropic properties. Curr Opin Colloid In 2004, 8 (6), 469-479, DOI 10.1016/j.cocis.2004.01.005.

3. Berchel, M.; Le Gall, T.; Lozach, O.; Haelters, J. P.; Montier, T.; Jaffres, P. A., Lipophosphoramidate-based bipolar amphiphiles: their syntheses and transfection properties. Org Biomol Chem 2016, 14 (10), 2846-2853, DOI 10.1039/c5ob02512e.

4. Le Gall, T.; Barbeau, J.; Barrier, S.; Berchel, M.; Lemiegre, L.; Jeftic, J.; Meriadec, C.; Artzner, F.; Gill, D. R.; Hyde, S. C.; Ferec, C.; Lehn, P.; Jaffres, P. A.; Benvegnu, T.; Montier, T., Effects of a Novel Archaeal Tetraether-Based Colipid on the In Vivo Gene Transfer Activity of Two Cationic Amphiphiles. Mol Pharmaceut 2014, 11 (9), 2973-2988, DOI 10.1021/mp4006276.

5. Benvegnu, T.; Rethore, G.; Brard, M.; Richter, W.; Plusquellec, D., Archaeosomes based on novel synthetic tetraether-type lipids for the development of oral delivery systems. Chem Commun 2005, (44), 5536-5538, DOI 10.1039/B511440C.

6. Cornell, B. A.; BraachMaksvytis, V. L. B.; King, L. G.; Osman, P. D. J.; Raguse, B.; Wieczorek, L.; Pace, R. J., A biosensor that uses ion-channel switches. Nature 1997, 387 (6633), 580-583, DOI $10.1038 / 42432$.

7. $\quad$ Nuraje, N.; Bai, H. Y.; Su, K., Bolaamphiphilic molecules: Assembly and applications. Prog Polym Sci 2013, 38 (2), 302-343, DOI 10.1016/j.progpolymsci.2012.09.003. 
8. Benvegnu, T.; Plusquellec, D.; Lemiegre, L., Surfactants from renewable sources: Synthesis and applications. In Monomers, polymers and composites from renewable resources, Belgacem, M. N.; Gandini, A., Eds. Elsevier: Oxford, UK, 2008; p 560.

9. Grinberg, S.; Kipnis, N.; Linder, C.; Kolot, V.; Heldman, E., Asymmetric bolaamphiphiles from vernonia oil designed for drug delivery. Eur. J. Lipid Sci. Technol. 2010, 112 (1), 137-151, DOI 10.1002/ejlt.200900107.

10. Ewonkem, M. B.; Grinberg, S.; Lemcoff, G.; Shaubi, E.; Linder, C.; Heldman, E., Newly synthesized bolaamphiphiles from castor oil and their aggregated morphologies for potential use in drug delivery. Tetrahedron 2015, 71 (45), 8557-8571, DOI 10.1016/j.tet.2015.09.031.

11. Shimizu, T.; Masuda, M., Stereochemical effect of even-odd connecting links on supramolecular assemblies made of 1-glucosamide bolaamphiphiles. J Am Chem Soc 1997, 119 (12), 2812-2818, DOI 10.1021/Ja961226y.

12. Guilbot, J.; Benvegnu, T.; Legros, N.; Plusquellec, D.; Dedieu, J. C.; Gulik, A., Efficient synthesis of unsymmetrical bolaamphiphiles for spontaneous formation of vesicles and disks with a transmembrane organization. Langmuir 2001, 17 (3), 613-618, DOI 10.1021/La000892g.

13. Benvegnu, T.; Lecollinet, G.; Guilbot, J.; Roussel, M.; Brard, M.; Plusquellec, D., Novel bolaamphiphiles with saccharide polar headgroups: synthesis and supramolecular self-assembilies. Polym Int 2003, 52 (4), 500-506, DOI 10.1002/pi.1056.

14. Garamus, V. M.; Milkereit, G.; Gerber, S.; Vill, V., Micellar structure of a sugar based bolaamphiphile in pure solution and destabilizing effects in mixtures of glycolipids. Chem Phys Lett 2004, 392 (1-3), 105-109, DOI 10.1016/j.cplett.2004.05.058.

15. Masuda, M.; Shimizu, T., Lipid nanotubes and microtubes: Experimental evidence for unsymmetrical monolayer membrane formation from unsymmetrical bolaamphiphiles. Langmuir 2004, 20 (14), 5969-5977, DOI 10.1021/La049085y.

16. Gerber, S.; Garamus, V. M.; Milkereit, G.; Vill, V., Mixed micelles formed by SDS and a bolaamphiphile with carbohydrate headgroups. Langmuir 2005, 21 (15), 6707-6711, DOI 10.1021/la050439a.

17. Roussel, M.; Lognone, V.; Plusquellec, D.; Benvegnu, T., Monolayer lipid membrane-forming dissymmetrical bolaamphiphiles derived from alginate oligosaccharides. Chem. Commun. 2006, (34), 3622-3624, DOI 10.1039/b607368a.

18. Bize, C.; Garrigues, J. C.; Corbet, J. P.; Rico-Lattes, I.; Blanzat, M., Bioactive Formulations with Sugar-Derived Surfactants: A New Approach for Photoprotection and Controlled Release of Promethazine. Chemphyschem 2013, 14 (6), 1126-1131, DOI 10.1002/cphc.201200932.

19. Shchelik, I. S.; Sebyakin, Y. L., Design and synthesis of an octavalent bolaamphiphile with terminal lactose residues. Russ J Org Chem+ 2015, 51 (12), 1717-1720, DOI $10.1134 / \mathrm{S} 1070428015120088$.

20. Zhan, C. L.; Gao, P.; Liu, M. H., Self-assembled helical spherical-nanotubes from an L-glutamic acid based bolaamphiphilic low molecular mass organogelator. Chem. Commun. 2005, (4), 462-464, DOI 10.1039/b413259a.

21. Ambrosi, M.; Fratini, E.; Alfredsson, V.; Ninham, B. W.; Giorgi, R.; Lo Nostro, P.; Baglioni, P., Nanotubes from a vitamin C-based bolaamphiphile. J Am Chem Soc 2006, 128 (22), 7209-7214, DOI 10.1021/ja057730x.

22. Hu, B. B.; Yuan, Y.; Zhou, X. P.; Li, S. M., Synthesis and properties of a novel bolaamphiphile surfactant derived from proline. Chinese Chem Lett 2016, 27 (3), 447-450, DOI 10.1016/j.cclet.2015.12.019.

23. Naess, S. N.; Elgsaeter, A.; Foss, B. J.; Li, B. J.; Sliwka, H. R.; Partali, V.; Melo, T. B.; Naqvi, K. R., Hydrophilic carotenoids: Surface properties and aggregation of crocin as a biosurfactant. Helv Chim Acta 2006, 89 (1), 45-53, DOI 10.1002/hlca.200690011.

24. Van Bogaert, I. N. A.; Saerens, K.; De Muynck, C.; Develter, D.; Soetaert, W.; Vandamme, E. J., Microbial production and application of sophorolipids. Appl Microbiol Biot 2007, 76 (1), 23-34, DOI 10.1007/s00253-007-0988-7.

25. Van Bogaert, I. N. A.; Zhang, J. X.; Soetaert, W., Microbial synthesis of sophorolipids. Process Biochem. 2011, 46 (4), 821-833, DOI 10.1016/j.procbio.2011.01.010.

26. Morya, V. K.; Ahn, C.; Jeon, S.; Kim, E. K., Medicinal and cosmetic potentials of sophorolipids.

Mini-Rev. Med. Chem. 2013, 13 (12), 1761-1768, DOI 10.2174/13895575113139990002. 
27. Delbeke, E. I. P.; Movsisyan, M.; Van Geem, K. M.; Stevens, C. V., Chemical and enzymatic modification of sophorolipids. Green Chem 2016, 18, 76-104, DOI 10.1039/C5GC02187A.

28. Baccile, N.; Nassif, N.; Malfatti, L.; Van Bogaert, I. N. A.; Soetaert, W.; Pehau-Arnaudet, G.; Babonneau, F., Sophorolipids: a yeast-derived glycolipid as greener structure directing agents for selfassembled nanomaterials. Green Chem. 2010, 12 (9), 1564-1567, DOI 10.1039/C0gc00163e.

29. Baccile, N.; Babonneau, F.; Jestin, J.; Pehau-Arnaudet, G.; Van Bogaert, I., Unusual, pHinduced, self-assembly of sophorolipid biosurfactants. ACS Nano 2012, 6 (6), 4763-4776, DOI $10.1021 / \mathrm{nn} 204911 \mathrm{k}$.

30. Baccile, N.; Pedersen, J. S.; Pehau-Arnaudet, G.; Van Bogaert, I. N. A., Surface charge of acidic sophorolipid micelles: effect of base and time. Soft Matter 2013, 9 (19), 4911-4922, DOI 10.1039/c3sm50160d.

31. Cuvier, A. S.; Berton, J.; Stevens, C. V.; Fadda, G. C.; Babonneau, F.; Van Bogaert, I. N. A.; Soetaert, W.; Pehau-Arnaudet, G.; Baccile, N., pH-triggered formation of nanoribbons from yeastderived glycolipid biosurfactants. Soft Matter 2014, 10 (22), 3950-3959, DOI 10.1039/c4sm00111g.

32. Cuvier, A. S.; Babonneau, F.; Berton, J.; Stevens, C. V.; Fadda, G. C.; Genois, I.; Le Griel, P.; Pehau-Arnaudet, G.; Baccile, N., Synthesis of uniform, monodisperse, sophorolipid twisted ribbons. Chem. - Asian J. 2015, 10 (11), 2419-2426, DOI 10.1002/asia.201500693.

33. Van Bogaert, I. N. A.; Buyst, D.; Martins, J. C.; Roelants, S. L. K. W.; Soetaert, W. K., Synthesis of bolaform biosurfactants by an engineered Starmerella bombicola yeast. Biotechnol. Bioeng. 2016, DOI 10.1002/bit.26032.

34. Van Renterghem, L.; Roelants, S. L. K. W.; Baccile, N.; Uyttersprot, K.; Taelman, M. C.; Everaert, B.; Mincke, S.; Ledegen, S.; Debrouwer, S.; Scholtens, K.; Stevens, C. V.; Soetaert, W., From lab to market: An integrated bioprocess design approach for new-to-nature biosurfactants produced by Starmerella bombicola. Biotechnol. Bioeng. 2018, DOI 10.1002/bit.26539.

35. Delbeke, E. I. P.; Roman, B. I.; Marin, G. B.; Van Geem, K. M.; Stevens, C. V., A new class of antimicrobial biosurfactants: quaternary ammonium sophorolipids. Green Chem 2015, 17 (6), 3373 3377, DOI 10.1039/c5gc00120j.

36. Delbeke, E. I. P.; Lozach, O.; Le Gall, T.; Berchel, M.; Montier, T.; Jaffres, P. A.; Van Geem, K. M.; Stevens, C. V., Evaluation of the transfection efficacies of quaternary ammonium salts prepared from sophorolipids. Org. Biomol. Chem. 2016, 14 (15), 3744-3751, DOI 10.1039/c6ob00241b.

37. Delbeke, E. Innovative sophorolipid analogues with tailor-made physico-chemical and biological properties. Ghent University, Ghent, 2016.

38. Delbeke, E. I. P.; Roelants, S. L. K. W.; Matthijs, N.; Everaert, B.; Soetaert, W.; Coenye, T.; Van Geem, K. M.; Stevens, C. V., Sophorolipid Amine Oxide Production by a Combination of Fermentation Scale-up and Chemical Modification. Industrial \& Engineering Chemistry Research 2016, 55 (27), 7273-7281, DOI 10.1021/acs.iecr.6b00629.

39. (CLSI), C. a. L. S. I., Performance Standards for Antimicrobial Susceptibility Testing. Twentieth second Informational Supplement M100-S22. Wayne: PA, USA, 2012.

40. $\quad$ Le Corre, S. S.; Berchel, M.; Le Gall, T.; Haelters, J. P.; Lehn, P.; Montier, T.; Jaffres, P. A., Cationic Trialkylphosphates: Synthesis and Transfection Efficacies Compared to Phosphoramidate Analogues. Eur J Org Chem 2014, 8041-8048, DOI 10.1002/ejoc.201403103

41. Fraix, A.; Le Gall, T.; Berchel, M.; Denis, C.; Lehn, P.; Montier, T.; Jaffres, P. A., Cationic lipophosphoramidates with two disulfide motifs: synthesis, behaviour in reductive media and gene transfection activity. Org. Biomol. Chem. 2013, 11 (10), 1650-1658, DOI 10.1039/c3ob27261c.

42. Pernot, P.; Round, A.; Barrett, R.; Antolinos, A. D.; Gobbo, A.; Gordon, E.; Huet, J.; Kieffer, J.; Lentini, M.; Mattenet, M.; Morawe, C.; Mueller-Dieckmann, C.; Ohlsson, S.; Schmid, W.; Surr, J.; Theveneau, P.; Zerrad, L.; McSweeney, S., Upgraded ESRF BM29 beamline for SAXS on macromolecules in solution. $J$ Synchrotron Radiat 2013, 20, 660-664, DOI 10.1107/S0909049513010431.

43. Round, A.; Felisaz, F.; Fodinger, L.; Gobbo, A.; Huet, J.; Villard, C.; Blanchet, C. E.; Pernot, P.; McSweeney, S.; Roessle, M.; Svergun, D. I.; Cipriani, F., BioSAXS Sample Changer: a robotic sample changer for rapid and reliable high-throughput X-ray solution scattering experiments. Acta Crystallogr D 2015, 71, 67-75, DOI 10.1107/S1399004714026959. 
44. Ashiotis, G.; Deschildre, A.; Nawaz, Z.; Wright, J. P.; Karkoulis, D.; Picca, F. E.; Kieffer, J., The fast azimuthal integration Python library: pyFAI. J Appl Crystallogr 2015, 48, 510-519, DOI 10.1107/S1600576715004306.

45. De Vos, N. Development of new ionic liquids based on a 7-aza- or 7-thiabicyclo[2.2.1] heptane skeleton. Ghent University, Ghent, 2013.

46. De Coen, L. M.; Roman, B. I.; Movsisyan, M.; D'hont, B.; De Vos, N.; Van Hecke, K.; Stevens, C. V., A new class of bicyclic dicationic salts based on the 7-azoniabicyclo [2.2.1] heptane scaffold. Tetrahedron Lett 2017, 58 (37), 3612-3615, DOI 10.1016/j.tetlet.2017.07.107.

47. Delbeke, E. I. P.; Everaert, J.; Uitterhaegen, E.; Verweire, S.; Verlee, A.; Talou, T.; Soetaert, W.; Van Bogaert, I. N. A.; Stevens, C. V., Petroselinic acid purification and its use for the fermentation of new sophorolipids. Amb Express 2016, 6, DOI 10.1186/s13568-016-0199-7.

48. Peng, Y.; Totsingan, F.; Meier, M. A. R.; Steinmann, M.; Wurm, F.; Koh, A.; Gross, R. A., Sophorolipids: Expanding structural diversity by ring-opening cross-metathesis. Eur J Lipid Sci Tech 2015, 117, 217-228, DOI 10.1002/ej1t.201400466.

49. Brackman, G.; Cos, P.; Maes, L.; Nelis, H. J.; Coenye, T., Quorum Sensing Inhibitors Increase the Susceptibility of Bacterial Biofilms to Antibiotics In Vitro and In Vivo. Antimicrob. Agents Chemother. 2011, 55 (6), 2655-2661, DOI 10.1128/Aac.00045-11.

50. Perche, F.; Benvegnu, T.; Berchel, M.; Lebegue, L.; Pichon, C.; Jaffres, P. A.; Midoux, P., Enhancement of dendritic cells transfection in vivo and of vaccination against B16F10 melanoma with mannosylated histidylated lipopolyplexes loaded with tumor antigen messenger RNA. NanomedNanotechnol 2011, 7 (4), 445-453, DOI 10.1016/j.nano.2010.12.010.

51. Leung, A. K. K.; Tam, Y. Y. C.; Cullis, P. R., Lipid Nanoparticles for Short Interfering RNA Delivery. Adv Genet 2014, 88, 71-110, DOI 10.1016/B978-0-12-800148-6.00004-3.

52. Guo, X.; Huang, L., Recent Advances in Nonviral Vectors for Gene Delivery. Accounts Chem Res 2012, 45 (7), 971-979, DOI 10.1021/ar200151m.

53. Berchel, M.; Le Gall, T.; Couthon-Gourves, H.; Haelters, J. P.; Montier, T.; Midoux, P.; Lehn, P.; Jaffres, P. A., Lipophosphonate/lipophosphoramidates: A family of synthetic vectors efficient for gene delivery. Biochimie 2012, 94 (1), 33-41, DOI 10.1016/j.biochi.2011.07.026.

54. Montier, T.; Benvegnu, T.; Jaffres, P. A.; Yaouanc, J. J.; Lehn, P., Progress in Cationic LipidMediated Gene Transfection: A Series of Bio-Inspired Lipids as an Example. Curr Gene Ther 2008, 8 (5), 296-312, DOI 10.2174/156652308786070989.

55. Liu, D. L.; Qiao, W. H.; Li, Z. S.; Chen, Y. X.; Cui, X. Y.; Li, K.; Yu, L. H.; Yan, K. L.; Zhu, L. M.; Guo, Y. L.; Cheng, L. B., Structure-function relationship research of glycerol backbone-based cationic lipids for gene delivery. Chem Biol Drug Des 2008, 71 (4), 336-344, DOI 10.1111/j.1747.0285.2008.00644.x.

56. Le Gall, T.; Loizeau, D.; Picquet, E.; Carmoy, N.; Yaouanc, J. J.; Burel-Deschamps, L.; Delepine, P.; Giamarchi, P.; Jaffres, P. A.; Lehn, P.; Montier, T., A Novel Cationic Lipophosphoramide with Diunsaturated Lipid Chains: Synthesis, Physicochemical Properties, and Transfection Activities. $J$ Med Chem 2010, 53 (4), 1496-1508, DOI 10.1021/jm900897a.

57. Lindberg, M. F.; Carmoy, N.; Le Gall, T.; Fraix, A.; Berchel, M.; Lorilleux, C.; CouthonGourves, H.; Bellaud, P.; Fautrel, A.; Jaffres, P. A.; Lehn, P.; Montier, T., The gene transfection properties of a lipophosphoramidate derivative with two phytanyl chains. Biomaterials 2012, 33 (26), 6240-6253, DOI 10.1016/j.biomaterials.2012.05.014.

58. Glatter, O.; Kratky, O.,Small Angle X-ray Scattering. Academic Press: New York, 1982.

59. Tanford, C.,The hydrophobic effect: Formation of micelles and biological membranes. Wiley: New York, 1980.

60. Stradner, A.; Glatter, O.; Schurtenberger, P., A hexanol-induced sphere-to-flexible cylinder transition in aqueous alkyl polyglucoside solutions. Langmuir 2000, 16 (12), 5354-5364, DOI 10.1021/la991679r.

61. Zhang, R. T.; Marone, P. A.; Thiyagarajan, P.; Tiede, D. M., Structure and molecular fluctuations of n-alkyl-beta-D-glucopyranoside micelles determined by X-ray and neutron scattering. Langmuir 1999, 15 (22), 7510-7519, DOI 10.1021/la990076c.

62. $\quad$ Milkereit, G.; Garamus, V. M.; Veermans, K.; Willumeit, R.; Vill, V., Structures of micelles formed by synthetic alkyl glycosides with unsaturated alkyl chains. J. Colloid Interface Sci. 2005, 284 (2), 704-713, DOI 10.1016/j.jcis.2004.10.039. 
63. Cuvier, A. S.; Babonneau, F.; Berton, J.; Stevens, C. V.; Fadda, G. C.; Genois, I.; Le Griel, P.; Pehau-Arnaudet, G.; Baccile, N., Synthesis of Uniform, Monodisperse, Sophorolipid Twisted Ribbons. Chem-Asian J 2015, 10 (11), 2419-2426, DOI 10.1002/asia.201500693.

64. Penfold, J.; Chen, M. L.; Thomas, R. K.; Dong, C. C.; Smyth, T. J. P.; Perfumo, A.; Marchant, R.; Banat, I. M.; Stevenson, P.; Parry, A.; Tucker, I.; Grillo, I., Solution Self-Assembly of the Sophorolipid Biosurfactant and Its Mixture with Anionic Surfactant Sodium Dodecyl Benzene Sulfonate. Langmuir 2011, 27 (14), 8867-8877, DOI 10.1021/la201661y.

65. Israelachvili, J. N.; Mitchell, D. J.; Ninham, B. W., Theory of Self-Assembly of Lipid Bilayers and Vesicles. Biochim Biophys Acta 1977, 470 (2), 185-201, DOI 10.1016/0005-2736(77)90099-2.

66. Israelachvili, J. N.; Mitchell, D. J.; Ninham, B. W., Theory of Self-Assembly of Hydrocarbon Amphiphiles into Micelles and Bilayers. J Chem Soc Farad T 2 1976, 72, 1525-1568, DOI 10.1039/f29767201525.

67. Jung, J. H.; Shinkai, S.; Shimizu, T., Spectral characterization of self-assemblies of aldopyranoside amphiphilic gelators: What is the essential structural difference between simple amphiphiles and bolaamphiphiles? Chem-Eur $J$ 2002, 8 (12), 2684-2690, DOI 10.1002/15213765(20020617)8:12<2684::Aid-Chem2684>3.0.Co;2-Z. 


\section{For Table of Contents Use Only}

Table of Contents graphic:

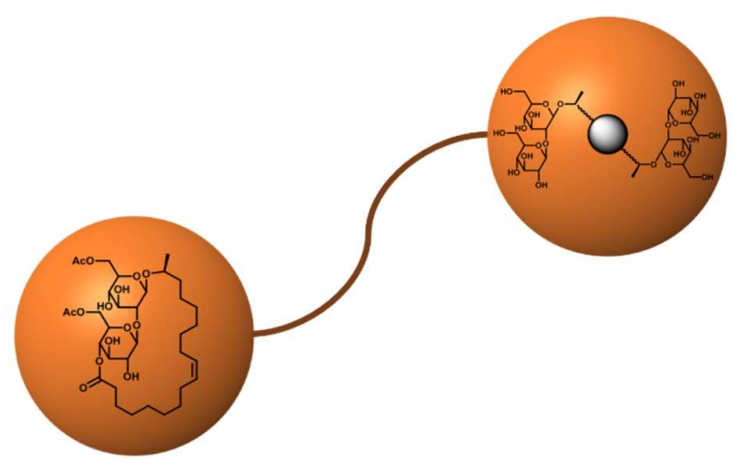

Sophorolipids, renewable biosurfactants, are used for the synthesis of innovative bolaamphiphilic compounds for application in the medical sector. 